\title{
Gauge drivers for the generalized harmonic Einstein equations
}

\author{
Lee Lindblom, ${ }^{1}$ Keith D. Matthews, ${ }^{1}$ Oliver Rinne, ${ }^{1,2,3}$ and Mark A. Scheel ${ }^{1}$ \\ ${ }^{1}$ Theoretical Astrophysics 130-33, California Institute of Technology, Pasadena, California 91125, USA \\ ${ }^{2}$ Department of Applied Mathematics and Theoretical Physics, Centre for Mathematical Sciences, \\ Wilberforce Road, Cambridge CB3 OWA, United Kingdom \\ ${ }^{3}$ King's College, Cambridge CB2 1ST, United Kingdom \\ (Received 13 November 2007; published 1 April 2008)
}

The generalized harmonic representation of Einstein's equations is manifestly hyperbolic for a large class of gauge conditions. Unfortunately most of the useful gauges developed over the past several decades by the numerical relativity community are incompatible with the hyperbolicity of the equations in this form. This paper presents a new method of imposing gauge conditions that preserves hyperbolicity for a much wider class of conditions, including as special cases many of the standard ones used in numerical relativity: e.g., $K$ freezing, $\Gamma$ freezing, Bona-Massó slicing, conformal $\Gamma$ drivers, etc. Analytical and numerical results are presented which test the stability and the effectiveness of this new gauge-driver evolution system.

DOI: 10.1103/PhysRevD.77.084001

\section{INTRODUCTION}

The gauge (or coordinate) degrees of freedom in the generalized harmonic $(\mathrm{GH})$ form of the Einstein equations are determined by specifying the gauge source functions $H^{a}$. These functions are defined as the results of the covariant scalar-wave operator acting on each of the spacetime coordinates $x^{a}$ :

$$
H^{a}=\nabla^{c} \nabla_{c} x^{a} .
$$

The GH form of the Einstein equations can be represented (somewhat abstractly) as

$$
\psi^{c d} \partial_{c} \partial_{d} \psi_{a b}+\partial_{a} H_{b}+\partial_{b} H_{a}=Q_{a b}(H, \psi, \partial \psi),
$$

where $\psi_{a b}$ is the spacetime metric, $H_{a}=\psi_{a b} H^{b}$, and $Q_{a b}$ represents lower order terms that depend on $H_{a}$, the metric, and its first derivatives.

The GH form of the Einstein equations is manifestly hyperbolic whenever $H_{a}$ is specified as an explicit function of the coordinates and the metric: $H_{a}=H_{a}(x, \psi)$. In this case the terms $\partial_{a} H_{b}$ that appear in Eq. (2) contain at most first derivatives of the metric. The Einstein equations become, therefore, a set of second-order wave equations for each component of the spacetime metric:

$$
\psi^{c d} \partial_{c} \partial_{d} \psi_{a b}=\hat{Q}_{a b}(x, \psi, \partial \psi) .
$$

Thus the Einstein equations are manifestly hyperbolic for any $H_{a}=H_{a}(x, \psi)$.

Most of the useful gauge conditions developed by the numerical relativity community over the past several decades cannot, unfortunately, be expressed in the simple form $H_{a}=H_{a}(x, \psi)$ (unless the full spacetime metric $\psi_{a b}=$ $\psi_{a b}[x]$ is known a priori). Many of these conditions (e.g., maximal slicing or $\Gamma$ drivers) would require gauge source functions that depend on the spacetime metric and its first derivatives: $H_{a}=H_{a}(x, \psi, \partial \psi)$. In this case the terms $\partial_{a} H_{b}$ in Eq. (2) depend on the second derivatives
PACS numbers: 04.25.D-, 02.60.Cb, 04.20.Cv, 04.25.dg

of the metric, $\psi_{a b}$, and this (generically) destroys the hyperbolicity of the system.

Pretorius [1-3] proposed a way to expand significantly the class of allowed gauge conditions, by elevating $H_{a}$ to the status of an independent dynamical field. A separate gauge-driver equation is introduced to evolve $H_{a}$, for example,

$$
\nabla^{c} \nabla_{c} H_{a}=Q_{a}(x, H, \partial H, \psi, \partial \psi),
$$

where $\nabla^{c} \nabla_{c} H_{a}$ denotes the wave operator ${ }^{1}$ acting on $H_{a}$. This gauge-driver equation is solved together with the GH Einstein equations to determine $\psi_{a b}$ and $H_{a}$ simultaneously. In the combined evolution system, consisting of Eqs. (2) and (4), the $\partial_{a} H_{b}$ terms in Eq. (2) are now lowerderivative terms that do not affect the hyperbolicity of the system. Thus the combined GH Einstein plus gauge-driver system is manifestly hyperbolic so long as $Q_{a}$ on the right in Eq. (4) depends only on the fields and their first derivatives: $Q_{a}=Q_{a}(x, H, \partial H, \psi, \partial \psi)$. Each of the solutions, $H_{a}=H_{a}(x)$, to these gauge-driver equations is a gauge condition. So the gauge-driver system provides a way for $H_{a}$ to be determined by the metric and its derivatives in a flexible way without destroying the hyperbolicity of the GH Einstein equations.

Pretorius used a particular gauge-driver equation of this form to determine $H_{t}$ in his ground breaking binary blackhole simulations:

$$
\nabla^{c} \nabla_{c} H_{t}=\xi_{1}(1-N) N^{-p}+\xi_{2}\left(\partial_{t} H_{t}-N^{k} \partial_{k} H_{t}\right) N^{-1},
$$

where in this case $\nabla^{c} \nabla_{c}$ is the covariant scalar-wave operator, $N$ is the lapse, $N^{k}$ is the shift, and $\xi_{1}, \xi_{2}$, and $p$ are constants. For suitable choices of these parameters,

\footnotetext{
${ }^{1}$ We define exactly what we mean by this wave operator in Sec. II B.
} 
Pretorius found this system to be quite effective in preventing the lapse from "collapsing" toward zero as the system evolves. Solutions to this gauge driver do not correspond to any of the traditional gauge conditions of numerical relativity as far as we know.

In this paper we introduce a new class of gauge-driver equations that are general enough to provide implementations of (almost) all of the standard gauge conditions used by the numerical relativity community. This is done by choosing an appropriate "source" term $Q_{a}$ for the right side of Eq. (4). The idea is to choose $Q_{a}$ so that solutions $H_{a}$ evolve quickly toward a target gauge source function $F_{a}$. This $F_{a}$ is chosen so that strict equality $H_{a}=F_{a}$ corresponds exactly to the gauge condition of interest to us. We limit $F_{a}$ only by assuming that it depends on the spacetime metric and its first (but not second) derivatives: $F_{a}=F_{a}(x, \psi, \partial \psi)$. These new gauge-driver equations are introduced in Sec. II, and we show there that the combined GH Einstein plus gauge-driver system is symmetric hyperbolic for any target gauge source function of this allowed form. In Sec. III we present the target gauge functions $F_{a}$ corresponding to (many of) the gauge conditions commonly used by the numerical relativity community, including maximal slicing, $K$ freezing, Bona-Massó slicing, conformal $\Gamma$ freezing, and conformal $\Gamma$ drivers. In Sec. IV we use analytical methods to analyze the solutions of the new gauge-driver system. We show in particular that $H_{a}$ approaches any (time-independent) $F_{a}$ exponentially for evolutions of the gauge-driver equations on flat space. We also demonstrate the effectiveness of the coupled GH Einstein and gauge-driver system for the case of small perturbations of flat space using Bona-Massó slicing and one of the conformal $\Gamma$-driver conditions. In Sec. $V$ we show the effectiveness and stability of our implementation of a particular choice of Bona-Massó slicing and conformal $\Gamma$-driver condition using numerical solutions of the full nonlinear equations for perturbed single black-hole spacetimes. We summarize and discuss these various results in Sec. VI.

\section{GAUGE-DRIVER EQUATIONS}

We begin this section by deriving a system of gaugedriver equations in Sec. II A, and then constructing a general first-order representation of these equations in Sec. II B. We derive the characteristic fields for this system and their associated speeds in Sec. II C, and show that the coupled gauge-driver and GH Einstein system is symmetric hyperbolic. We analyze the constraints in Sec. II D, and derive constraint preserving boundary conditions for the gauge-driver fields in Sec. IIE.

\section{A. Motivation}

In this section we provide some motivation for our choice of gauge-driver equation. We consider first the case of the gauge-driver $\nabla^{c} \nabla_{c} H_{a}=Q_{a}$ acting on a fixed flat-space background. The idea is to choose $Q_{a}$ so that the solutions, $H_{a}$, to this equation quickly approach the desired target gauge source function $F_{a}$. If $F_{a}$ were constant in space and time, there would be a fairly obvious and simple choice:

$$
\nabla^{c} \nabla_{c} H_{a}=Q_{a}=\mu^{2}\left(H_{a}-F_{a}\right)+2 \mu \partial_{t} H_{a},
$$

where $\mu$ is a freely specifiable constant. If $H_{a}$ like $F_{a}$ were independent of spatial position, the gauge-driver equation would be equivalent in this case to the ordinary differential equation,

$$
\partial_{t}^{2}\left(H_{a}-F_{a}\right)+2 \mu \partial_{t}\left(H_{a}-F_{a}\right)+\mu^{2}\left(H_{a}-F_{a}\right)=0,
$$

whose solution has the form $H_{a}(t)=F_{a}+\left[H_{a}(0)-\right.$ $\left.F_{a}\right] e^{-\mu t}$. A similar argument applied to the spatial Fourier transform of Eq. (6) shows that spatially inhomogeneous $H_{a}$ also approach $F_{a}$ exponentially in time. In this special case (i.e., spatially homogeneous and timeindependent $F_{a}$ ) the simple gauge driver has the desired behavior: all the solutions $H_{a}$ approach the target gauge source $F_{a}$ exponentially on the adjustable time scale $1 / \mu$.

This simple gauge driver, Eq. (6), fails unfortunately even in flat space if $F_{a}$ is a generic function of position. An easy way to see this is to assume that all the solutions $H_{a}$ do approach $F_{a}$ asymptotically as $t \rightarrow \infty$. Since $F_{a}$ and consequently $H_{a}$ are independent of time in this limit, Eq. (6) reduces to $\nabla^{k} \nabla_{k} H_{a}=0$, where $\nabla^{k} \nabla_{k} H_{a}$ represents the spatial Laplacian of $H_{a}$. But this is impossible because $\nabla^{k} \nabla_{k} F_{a} \neq 0$ for generic $F_{a}$. So (not surprisingly) the simple gauge driver fails in general.

This gauge driver can be modified in a fairly straightforward way, however, that corrects this problem. Define an auxiliary dynamical field $\theta_{a}$ :

$$
\partial_{t} \theta_{a}+\eta \theta_{a}=\nabla^{k} \nabla_{k} H_{a} .
$$

This equation can be integrated analytically to obtain an equivalent integral representation of $\theta_{a}$ :

$$
\theta_{a}=\theta_{a}(0) e^{-\eta t}+\int_{0}^{t} e^{-\eta\left(t-t^{\prime}\right)} \nabla^{k} \nabla_{k} H_{a}\left(t^{\prime}\right) d t^{\prime}
$$

Thus $\theta_{a}$ represents an exponentially weighted (in favor of times near $t$ ) time average of the past evolution of the term on the right side of Eq. (8). We can use this $\theta_{a}$ to construct an improved gauge driver:

$$
\nabla^{c} \nabla_{c} H_{a}=Q_{a}=\mu^{2}\left(H_{a}-F_{a}\right)+2 \mu \partial_{t} H_{a}+\eta \theta_{a} .
$$

If a solution to the improved gauge driver approaches a time-independent state, then Eq. (8) implies that $\eta \theta_{a}=$ $\nabla^{k} \nabla_{k} H_{a}$. Equation (10) reduces in this case to $0=$ $\mu^{2}\left(H_{a}-F_{a}\right)$. So the addition of the time-averaging field $\theta_{a}$ forces $H_{a}$ to approach $F_{a}$ in any time-independent state, even in the case of inhomogeneous $F_{a}$. The remainder of this paper is devoted to the analysis of this improved gauge 
driver, Eq. (10), suitably generalized for use in an arbitrary spacetime.

\section{B. First-order form}

We find it very useful to consider the first-order representations of evolution systems (such as our gauge driver) for a variety of reasons: from basic mathematical issues (such as the formulation of appropriate boundary conditions) to more practical stability issues with our code. This section develops a first-order representation of the gaugedriver system, suitably generalized for use in an arbitrary curved spacetime.

The gauge-driver system described above evolves $H_{a}$ through a wave equation of the form $\nabla^{c} \nabla_{c} H_{a}=Q_{a}$. In a general curved spacetime, we assume that $\nabla^{c} \nabla_{c} H_{a}$ represents the covariant wave operator that treats $H_{a}$ as a covector. This choice needs a bit of clarification, since the gauge source function $H_{a}$ is not actually a covector. One way of giving meaning to this equation is to use the gauge-driver system to determine a new field $\tilde{H}_{a}$ that does transform as a covector: $\nabla^{c} \nabla_{c} \tilde{H}_{a}=Q_{a}$. Then fix $H_{a}$ by setting $H_{a}=\tilde{H}_{a}$ in some particular coordinate frame. This construction is not covariant, but fixing coordinate conditions can never be completely covariant. An equivalent way to do this is to write out and impose the gauge-driver equation, $\nabla^{c} \nabla_{c} H_{a}=Q_{a}$, only in the special coordinate frame in which $H_{a}=\tilde{H}_{a}$. We adopt this second approach since it simplifies the notation somewhat. So throughout this paper the gauge-driver equation, $\nabla^{c} \nabla_{c} H_{a}=Q_{a}$, will only be imposed in some particular coordinate frame that we must specify. In our code we use a global Cartesian coordinate system, and we will always impose the gaugedriver equation in that frame.

Before we discuss the first-order form of the gaugedriver equations, we also need to examine the somewhat pathological covariant vector wave operator in more detail. This operator acting on $H_{a}$ (assumed here to be a covector as discussed above) can be written out more explicitly in the form

$$
\begin{aligned}
\nabla^{c} \nabla_{c} H_{a}= & \psi^{b c} \partial_{b} \partial_{c} H_{a}-\Gamma^{b} \partial_{b} H_{a}-2 \psi^{b c} \Gamma^{d}{ }_{a c} \partial_{b} H_{d} \\
& +\left(R_{a}{ }^{b}-\partial_{a} \Gamma^{b}\right) H_{b},
\end{aligned}
$$

where $\Gamma_{b c}^{a}$ is the Christoffel connection, $\Gamma^{a}=\psi^{b c} \Gamma^{a}{ }_{b c}$, and $R_{a}{ }^{b}$ is the associated Ricci curvature. This wave operator is well behaved on a fixed background spacetime. However, the $H_{b} \partial_{a} \Gamma^{b}$ term includes second derivatives of the metric that would interfere with hyperbolicity, if it were coupled in a nontrivial way to the full Einstein equations. Fortunately this problem has a simple solution. Since we use the $\mathrm{GH}$ form of the Einstein equations, this term can be transformed into the more benign form, $-H_{b} \partial_{a} H^{b}$ (or if a more linear looking form is preferred $\Gamma_{b} \partial_{a} H^{b}$ ), using the gauge constraint $H^{a}=-\Gamma^{a}$ [4]. We regard the Ricci tensor $R_{a}{ }^{b}$ as being determined by the matter sources via the
Einstein equations; in particular, it does not contain any second derivatives of the metric. For notational convenience we introduce the quantity $W_{a}(H)$,

$$
W_{a}(H)=2 \psi^{b c} \Gamma_{a c}^{d} \partial_{b} H_{d}-\left(\partial_{a} H^{b}+R_{a}{ }^{b}\right) H_{b},
$$

that represents the parts of the vector wave operator that are not present in the scalar-wave operator. Our representation of the covariant vector wave operator is therefore given by

$$
\nabla^{c} \nabla_{c} H_{a}=\psi^{b c} \partial_{b} \partial_{c} H_{a}-\Gamma^{b} \partial_{b} H_{a}-W_{a}(H) .
$$

To represent this equation in first-order form, we introduce the usual additional first-order fields $\Pi_{a}^{H}$ and $\Phi_{i a}^{H}$ representing (up to the addition of constraints) the appropriate time and space derivatives of $H_{a}$, respectively,

$$
\begin{gathered}
\Pi_{a}^{H}=-t^{b} \partial_{b} H_{a}, \\
\Phi_{i a}^{H}=\partial_{i} H_{a} .
\end{gathered}
$$

Here (and throughout this paper) $t^{a}$ is the future directed unit normal to the $t=$ constant hypersurfaces; Latin indices $a$ through $h$ are spacetime indices and run from 0 to 3; and Latin indices $i$ through $n$ are spatial indices and run from 1 to 3 . We also define the spatial metric on the $t=$ constant hypersurfaces,

$$
g_{a b}=\psi_{a b}+t_{a} t_{b} .
$$

The covariant wave operator, $\nabla^{c} \nabla_{c} H_{a}$, can then be expressed in terms of these first-order field variables:

$$
\begin{aligned}
\nabla^{c} \nabla_{c} H_{a}= & t^{c} \partial_{c} \Pi_{a}^{H}+g^{i j} \partial_{i} \Phi_{j a}^{H}-t_{b} \Gamma^{b} \Pi_{a}^{H}-\Gamma^{i} \Phi_{i a}^{H} \\
& +\frac{1}{2} \Pi_{a}^{H} t^{b} t^{c} \Pi_{b c}+g^{i j} \Phi_{i a}^{H} t^{b} \Pi_{b j}-W_{a}(H),
\end{aligned}
$$

where $W_{a}(H)$ can be written as

$$
\begin{aligned}
W_{a}(H)= & \left(t_{a} \Pi_{b c}+g_{a}{ }^{i} \Phi_{i b c}\right) t^{b} \psi^{c d}\left(\Pi_{d}^{H}-t^{e} H_{d} H_{e}\right) \\
& +\left(t_{a} \Pi_{i b}+g_{a}{ }^{j} \Phi_{j i b}\right) \psi^{b c} g^{i k}\left(\Phi_{k c}^{H}+H_{k} H_{c}\right) \\
& -g^{i j} t^{b} \Pi_{i a} \Phi_{j b}^{H}+g^{i j} \psi^{b c} \Phi_{i a b} \Phi_{j c}^{H} \\
& -g^{i j} \Pi_{j}^{H}\left(\Pi_{a i}+t^{b} \Phi_{i a b}\right)-g^{i j} g^{k l} \Phi_{i k a} \Phi_{l j}^{H} \\
& +\left(t_{a} \Pi_{b}^{H}+g_{a}{ }^{i} \Phi_{i b}^{H}\right) \Gamma^{b}-R_{a}{ }^{b} H_{b} .
\end{aligned}
$$

We note that leaving out the $W_{a}(H)$ terms is equivalent to applying the covariant scalar-wave operator to each component of $H_{a}$ in our special coordinate frame. We also note that the remaining $\Gamma^{b}$ terms that appear in the above equations are to be thought of as functions of the firstorder GH fields:

$$
\Gamma^{b}=\psi^{b c} t^{d} \Pi_{c d}+g^{i j} \psi^{b c} \Phi_{i j c}-\frac{1}{2} \psi^{c d}\left(t^{b} \Pi_{c d}+g^{b i} \Phi_{i c d}\right) .
$$

The representation of wave equations of this type in first-order form is well understood (see e.g., Refs. [4,5]); the result for our gauge-driver equation is 


$$
\begin{aligned}
& \partial_{t} H_{a}-\left(1+\gamma_{1}^{H}\right) N^{k} \partial_{k} H_{a}=-N \Pi_{a}^{H}-\gamma_{1}^{H} N^{k} \Phi_{k a}^{H}, \quad(20) \\
& \partial_{t} \Pi_{a}^{H}-N^{k} \partial_{k} \Pi_{a}^{H}+N g^{k i} \partial_{k} \Phi_{i a}^{H}-\gamma_{1}^{H} \gamma_{2}^{H} N^{k} \partial_{k} H_{a} \\
& \quad=-\gamma_{1}^{H} \gamma_{2}^{H} N^{k} \Phi_{k a}^{H}+N J^{k} \Phi_{k a}^{H}+N K \Pi_{a}^{H}+Q_{a}+N W_{a},
\end{aligned}
$$

$$
\begin{gathered}
\partial_{t} \Phi_{i a}^{H}-N^{k} \partial_{k} \Phi_{i a}^{H}+N \partial_{i} \Pi_{a}^{H}-\gamma_{2}^{H} N \partial_{i} H_{a} \\
=-\Pi_{a}^{H} \partial_{i} N+\Phi_{k a}^{H} \partial_{i} N^{k}-\gamma_{2}^{H} N \Phi_{i a}^{H} .
\end{gathered}
$$

The quantities $N, N^{k}$, and $g^{i j}$ that appear in these equations are the lapse, shift, and inverse spatial metric, respectively. The spatial metric $g_{i j}$ is defined by the usual three-plus-one representation of the spacetime metric:

$$
\begin{aligned}
d s^{2} & =\psi_{a b} d x^{a} d x^{b} \\
& =-N^{2} d t^{2}+g_{i j}\left(d x^{i}+N^{i} d t\right)\left(d x^{j}+N^{j} d t\right) .
\end{aligned}
$$

The auxiliary quantities $K$ and $J^{i}$ in Eq. (21) depend on the background spacetime geometry and can be written in terms of the first-order GH Einstein variables:

$$
\begin{gathered}
K=\frac{1}{2} g^{i j} \Pi_{i j}+g^{i j} t^{a} \Phi_{i j a}, \\
J^{i}=\left(g^{j k} g^{l i}-\frac{1}{2} g^{i j} g^{k l}\right) \Phi_{j k l}+\frac{1}{2} g^{i j} t^{a} t^{b} \Phi_{j a b} .
\end{gathered}
$$

The constants $\gamma_{1}^{H}$ and $\gamma_{2}^{H}$ are introduced (in analogy with the first-order GH Einstein system [4]) to allow us to control the growth of constraint violations, and to allow us to adjust one of the characteristic speeds of the system.

The quantity $Q_{a}$ in Eq. (21) is defined by a natural generalization of Eq. (10):

$$
\begin{aligned}
Q_{a}= & \mu_{1}^{2}\left(1-\xi_{1}\right) N\left(H_{a}-F_{a}\right)-2 \mu_{2}\left(1-\xi_{2}\right) N \Pi_{a}^{H} \\
& +\eta_{1} \theta_{a} .
\end{aligned}
$$

The differences between this expression and Eq. (10) are an overall factor of the lapse $N$ (to convert from coordinate time to proper time), the replacement of $\partial_{t} H_{a}$ by $\Pi_{a}^{H}$ (the first-order field representing $-t^{c} \partial_{c} H_{a}$ ), the introduction of independent damping parameters $\mu_{1}$ and $\mu_{2}$, and the introduction of new constant parameters $\xi_{1}$ and $\xi_{2}$. The purpose of these latter parameters, $\xi_{1}$ and $\xi_{2}$, is to move the damping terms (or fractions thereof) into the source for the time-averaging field $\theta_{a}$ [cf. Eq. (27) below], thus effectively replacing these terms by their time averages. We assume as before that $F_{a}$ is a given function of the fourmetric and its first derivatives: $F_{a}=F_{a}(x, \psi, \partial \psi)$.

The evolution equation for $\theta_{a}$ is chosen, in analogy with Eq. (8), to include as its source all the terms in Eq. (21) that do not vanish automatically in a time-independent state:

$$
\begin{aligned}
\partial_{t} \theta_{a}+\eta_{1} \theta_{a}= & 2 \mu_{2}\left[\xi_{3} N \Pi_{a}^{H}+\left(1-\xi_{3}\right)\left(1+\gamma_{1}^{H}\right) N^{k} \partial_{k} H_{a}\right] \\
& +N g^{k i} \partial_{k} \Phi_{i a}^{H}-N^{k} \partial_{k} \Pi_{a}^{H}-\gamma_{1}^{H} \gamma_{2}^{H} N^{k} \partial_{k} H_{a} \\
& -2 \mu_{2}\left(1-\xi_{3}\right) \gamma_{1}^{H} N^{k} \Phi_{k a}^{H}+\gamma_{1}^{H} \gamma_{2}^{H} N^{k} \Phi_{k a}^{H} \\
& -N K \Pi_{a}^{H}-N J^{i} \Phi_{i a}^{H}-N W_{a} \\
& +\mu_{1}^{2} \xi_{1} N\left(H_{a}-F_{a}\right)-2 \mu_{2} \xi_{2} N \Pi_{a}^{H} .
\end{aligned}
$$

The $\xi_{3}$ parameter is introduced to add a multiple of $\partial_{t} H_{a}$ to the source of the time-averaging field. We use Eq. (20) to reexpress this $\partial_{t} H_{a}$ as the terms proportional to $\xi_{3}$ that appear on the right side of Eq. (27). Assuming the system approaches a state in which $H_{a}$ becomes time independent, then $\theta_{a}$ exponentially approaches the time-independent limit of the terms on the right side of Eq. (27). These terms were chosen so that Eq. (21) then implies that $H_{a} \rightarrow F_{a}$ in this limit. Our choices for the parameters $\mu_{1}, \mu_{2}, \eta_{1}, \xi_{1}$, $\xi_{2}$, and $\xi_{3}$ that appear in Eqs. (26) and (27) will be guided by the stability analysis that we perform in Sec. IV.

\section{Characteristic fields}

The gauge-driver evolution Eqs. (20)-(22) and (27) comprise a first-order evolution system of the form

$$
\partial_{t} u^{\alpha}+A_{\beta}^{k \alpha} \partial_{k} u^{\beta}=B^{\alpha}
$$

for the fields $u^{\alpha}=\left\{H_{a}, \Pi_{a}^{H}, \Phi_{i a}^{H}, \theta_{a}\right\}$ (treating the spacetime metric for the moment as a fixed background field). The characteristic fields of such an evolution system are important for a number of reasons, including the formulation of outer boundary conditions and exchanging information across internal boundaries of the computational domain. The characteristic fields (in the direction of a unit spacelike covector $n_{k}$ ) are defined as the projections of the fields $u^{\alpha}$ onto the left eigenvectors of the characteristic matrix $n_{k} A_{\beta}^{k \alpha}$. For the gauge-driver system, these characteristic fields are

$$
\begin{gathered}
U_{a}^{H \pm}=\Pi_{a}^{H} \pm n^{i} \Phi_{i a}^{H}-\gamma_{2}^{H} H_{a}, \\
Z_{a}^{H 1}=H_{a}, \\
Z_{i a}^{H 2}=P_{i j} g^{j k} \Phi_{k a}^{H}, \\
Z_{a}^{H 3}=\theta_{a}+\Pi_{a}^{H}-2 \mu_{2}\left(1-\xi_{3}\right) H_{a},
\end{gathered}
$$

where $P_{i j} \equiv g_{i j}-n_{i} n_{j}$.

The eigenvalues associated with the characteristic fields are called the characteristic speeds of the system. For the gauge-driver system, the characteristic fields $U_{a}^{H \pm}$ have speeds $\pm N-n_{i} N^{i}, Z_{a}^{H 1}$ has speed $-\left(1+\gamma_{1}^{H}\right) n_{i} N^{i}, Z_{i a}^{H 2}$ has speed $-n_{i} N^{i}$, and $Z_{a}^{H 3}$ has speed zero.

The inverse transformation between dynamical fields and characteristic fields for our gauge-driver system is

$$
H_{a}=Z_{a}^{H 1},
$$




$$
\begin{gathered}
\Pi_{a}^{H}=\frac{1}{2}\left(U_{a}^{H+}+U_{a}^{H-}\right)+\gamma_{2}^{H} Z_{a}^{H 1}, \\
\Phi_{i a}^{H}=\frac{1}{2}\left(U_{a}^{H+}-U_{a}^{H-}\right) n_{i}+Z_{i a}^{H 2}, \\
\theta_{a}=Z_{a}^{H 3}-\frac{1}{2}\left(U_{a}^{H+}+U_{a}^{H-}\right)+2 \mu_{2}\left(1-\xi_{3}\right) Z_{a}^{H 1} \\
-\gamma_{2}^{H} Z_{a}^{H 1} .
\end{gathered}
$$

The existence of this inverse transformation shows that there is a one-to-one correspondence between the dynamical fields and the characteristic fields. This implies that the gauge-driver system is strongly hyperbolic.

A quasilinear evolution system, Eq. (28), is symmetric hyperbolic (a stronger condition than strong hyperbolicity) if there exists a positive definite metric $S_{\alpha \beta}$ (called a symmetrizer) on the space of fields, such that $S_{\alpha \gamma} A^{k \gamma}{ }_{\beta}=$ $S_{\beta \gamma} A^{k \gamma}{ }_{\alpha}$. The gauge-driver system, Eqs. (20)-(22) and (27), does have such a symmetrizer:

$$
\begin{aligned}
d S^{2}= & S_{\alpha \beta} d u^{\alpha} d u^{\beta} \\
= & \sum_{a}\left\{\Lambda_{a}^{2} d H_{a}^{2}+\left[d \theta_{a}-2 \mu_{2}\left(1-\xi_{3}\right) d H_{a}+d \Pi_{a}^{H}\right]^{2}\right. \\
& \left.+g^{i j} d \Phi_{i a}^{H} d \Phi_{j a}^{H}+\left(d \Pi_{a}^{H}-\gamma_{2}^{H} d H_{a}\right)^{2}\right\},
\end{aligned}
$$

where $\Lambda_{a}$ are arbitrary (nonvanishing) constants. The gauge-driver system is therefore symmetric hyperbolic.

Up to this point the discussion has focused on the properties of the gauge-driver system, Eqs. (20)-(22) and (27), with the spacetime metric considered as a fixed background field. Our real interest of course is the case where the gauge-driver system is coupled to the GH Einstein system, Eq. (2). Thus we need to consider the properties of the combined evolution system having as dynamical fields the gauge-driver fields plus the $\mathrm{GH}$ Einstein system fields: $u^{\alpha}=\left\{H_{a}, \Pi_{a}^{H}, \Phi_{i a}^{H}, \theta_{a}, \psi_{a b}\right.$, $\left.\Pi_{a b}, \Phi_{i a b}\right\}$. The fields $\Pi_{a b}$ and $\Phi_{i a b}$ represent the first derivatives of the spacetime metric $\psi_{a b}$, as defined, for example, in Ref. [4]. We need to analyze the properties of the characteristic matrix $A^{k \alpha}{ }_{\beta}$ of this combined system to determine whether the full coupled system is hyperbolic.

We have shown above that the gauge-driver system, Eqs. (20)-(22) and (27), is symmetric hyperbolic if the spacetime metric is considered as a background field. Similarly, the first-order representation of the GH Einstein system [4] is symmetric hyperbolic if the gauge source function $H_{a}$ is considered as a background field. The characteristic matrix $A_{\beta}^{k \alpha}$ of the combined system is block diagonal, except for any cross terms that might arise if derivatives of the gauge-driver fields appear in evolution equations for the GH fields or vice versa. The only potential cross terms are as follows: the term $2 \partial_{(a} H_{b)}$ occurs in the GH Einstein equations (2), the quantities $K$ and $J^{i}$ in Eq. (21) depend on derivatives of the spacetime metric, and the target gauge source function $F_{a}$ which appears as part of $Q_{a}$ in Eq. (21) [see Eq. (26)] may include derivatives of the spacetime metric.

However, $\partial_{(a} H_{b)}$ can be rewritten in terms of the firstorder gauge-driver variables as

$$
\partial_{(a} H_{b)}=\Phi_{i(a}^{H} g_{b)}{ }^{i}+\Pi_{(a}^{H} t_{b)} .
$$

Likewise, $K$ and $J^{i}$ can be expressed as algebraic functions of the first-order $\mathrm{GH}$ fields $\psi_{a b}, \Pi_{a b}$, and $\Phi_{i a b}$; cf. Eqs. (24) and (25). Finally, the target gauge source function $F_{a}$ is assumed to be a function of the metric and its first derivatives, so it also can be written as an algebraic function of the first-order fields: $F_{a}=F_{a}(x, \psi, \Pi, \Phi)$. Thus all of these potential cross terms can be written as algebraic functions of the dynamical fields and do not contribute to the characteristic matrix $A_{\beta}^{k \alpha}$ at all.

The characteristic matrix of the combined evolution system is therefore block diagonal. It follows that the characteristic fields of the combined system are just the collection of unmodified characteristic fields from the separate systems. Similarly the matrix $S_{\alpha \beta}$ needed to symmetrize the full system is just the matrix whose diagonal blocks are the symmetrizers of the individual systems. It follows trivially that the combined GH Einstein and gaugedriver system is both strongly and symmetric hyperbolic.

\section{Constraints}

The basic gauge-driver evolution system, Eq. (4), has no fundamental constraints. However, by transforming the system to first-order form, Eqs. (20)-(22), we introduce a set of new constraints:

$$
\begin{gathered}
\mathcal{C}_{i a}^{H}=\partial_{i} H_{a}-\Phi_{i a}^{H}, \\
\mathcal{C}_{i j a}^{H}=2 \partial_{[i} \mathcal{C}_{j] a}^{H}=-2 \partial_{[i} \Phi_{j] a}^{H} .
\end{gathered}
$$

These constraints vanish, $\mathcal{C}_{i a}^{H}=\mathcal{C}_{i j a}^{H}=0$, if and only if a solution to the first-order system also represents a solution to the original second-order equation.

These constraints are determined by the values of the dynamical fields $H_{a}$ and $\Phi_{i a}^{H}$; therefore their time evolution is determined by the gauge-driver evolution system. It is straightforward to show that these constraints satisfy the evolution equations

$$
\begin{gathered}
\partial_{t} \mathcal{C}_{i a}^{H}-\left(1+\gamma_{1}^{H}\right) N^{k} \partial_{k} \mathcal{C}_{i a}^{H}=- \\
-\gamma_{2}^{H} N \mathcal{C}_{i a}^{H}-\gamma_{1}^{H} N^{k} \mathcal{C}_{k i a}^{H}, \\
\partial_{t} \mathcal{C}_{i j a}^{H}-N^{k} \partial_{k} \mathcal{C}_{i j a}^{H}=\partial_{i} N^{k} \mathcal{C}_{k j a}^{H}+\partial_{j} N^{k} \mathcal{C}_{i k a}^{H}-\gamma_{2}^{H} N \mathcal{C}_{i j a}^{H} \\
-2 \gamma_{2}^{H} \partial_{[i} N \mathcal{C}_{j] a}^{H},
\end{gathered}
$$

as a consequence of Eqs. (20)-(22).

The characteristic matrix of this constraint evolution system is diagonal, so the constraints are themselves char- 
acteristic fields of this system. The constraint $\mathcal{C}_{i a}^{H}$ propagates at the speed $-\left(1+\gamma_{1}^{H}\right) n_{k} N^{k}$, while $\mathcal{C}_{i j a}^{H}$ propagates at the speed $-n_{k} N^{k}$. This constraint evolution system is strongly (and also symmetric) hyperbolic.

The constraint evolution system is also homogeneous in the constraints; i.e., the right sides of Eqs. (41) and (42) are proportional to the constraints. This implies, for example, that these constraints will remain satisfied within the domain of dependence of the subset of the initial surface on which they are satisfied.

\section{E. Boundary conditions}

Boundary conditions are needed for any of the characteristic fields having incoming (i.e., negative) characteristic speeds on the boundary. Some of these boundary conditions can be determined by the need to prevent the influx of constraint violations, while others can be chosen to control the particular gauge condition being imposed at the boundary. In analogy with the scalar field system [5], the needed constraint preserving boundary conditions for this system are

$$
\begin{gathered}
d_{t} Z_{a}^{H 1}=D_{t} Z_{a}^{H 1}-\left(1+\gamma_{1}^{H}\right) n_{k} N^{k} n^{i} \mathcal{C}_{i a}^{H}, \\
d_{t} Z_{i a}^{H 2}=D_{t} Z_{i a}^{H 2}-n_{k} N^{k} P_{i}{ }^{j} n^{l} \mathcal{C}_{j l a}^{H},
\end{gathered}
$$

where $d_{t} Z_{a}^{H 1}=\partial_{t} H_{a}$ and $d_{t} Z_{i a}^{H 2}=P_{i}{ }^{k} \partial_{t} \Phi_{k a}^{H}$ represent the constraint field projections of the time derivatives of the dynamical fields, while $D_{t} Z_{a}^{H 1}$ and $D_{t} Z_{i a}^{H 2}$ represent the constraint field projections of the right sides of the evolution equations for these fields.

The characteristic fields $U_{a}^{H \pm}$ need boundary conditions whenever the corresponding speeds $v_{ \pm}= \pm N-n_{k} N^{k}$ are negative. Since $v_{-}<v_{+}$, typically the $U_{a}^{H-}$ mode is the one needing a boundary condition. The boundary condition on this field controls the incoming part of the gauge condition being imposed on the boundary. We often use a "freezing" boundary condition, $\partial_{t} U_{a}^{H-}=0$, or the boundary condition, $\partial_{t} U_{a}^{H-}=-\gamma_{2}^{H} \partial_{t} Z_{a}^{H 1}$. Ideally the boundary condition on the characteristic field $U_{a}^{H-}$ should be determined by the gauge condition that the driver equation is trying to enforce; however, at present we do not know how to do this.

\section{SPECIFIC GAUGE CONDITIONS}

The gauge-driver equations presented in Sec. II were designed to evolve the gauge source function, $H_{a}$, toward a target function $F_{a}=F_{a}(x, \psi, \partial \psi)$. The question of how well these equations accomplish this will be explored in Secs. IV and V. Here we focus on the issue of constructing target functions $F_{a}$ for particular gauge conditions used in numerical relativity.

Most of the gauge choices used by the numerical relativity community, including all the examples below, are expressed as conditions on the spacetime metric and its first (space and time) derivatives, so abstractly, all such gauge conditions can be written in the form $G_{a}(x, \psi, \partial \psi)=$ 0 . Whenever the GH Einstein constraints are satisfied, it follows from Eq. (1) that $H_{a}=-\Gamma_{a} \equiv-\Gamma_{a b c} \psi^{b c}$, where $\Gamma_{a b c}$ is the $4 \mathrm{D}$ Christoffel symbol. An appropriate target gauge source function $F_{a}$ is therefore given by

$$
F_{a}=-\Gamma_{a}-\rho G_{a}
$$

where $\rho$ is an arbitrary (nonvanishing) constant. When the constraints are satisfied, this equation implies that $H_{a}-$ $F_{a}=\rho G_{a}$. So if the gauge-driver system succeeds in driving $H_{a}-F_{a} \rightarrow 0$, it follows that $G_{a} \rightarrow 0$ as well for any $\rho \neq 0$. This $F_{a}$ has the general form assumed in the discussions of Sec. II, $F_{a}=F_{a}(x, \psi, \partial \psi)$, whenever $G_{a}$ has the form $G_{a}=G_{a}(x, \psi, \partial \psi)$. Therefore the gaugedriver system with this target $F_{a}$ should enforce the desired gauge condition $G_{a}=0$ asymptotically as the system evolves.

The numerical relativity community traditionally separates gauge conditions into those that determine the lapse $N$ (often called slicing conditions) and those that determine the spatial coordinates through the shift $N^{k}$. Expressing $\Gamma_{a}$ in terms of the three-plus-one representation of the spacetime metric, Eq. (23), reveals that different components of $\Gamma_{a}$ are naturally related to conditions on the lapse and shift, respectively,

$$
\begin{gathered}
\Gamma_{\hat{t}} \equiv t^{a} \Gamma_{a}=N^{-2}\left(\partial_{t} N-N^{i} \partial_{i} N\right)+K, \\
\Gamma_{i}=-N^{-2} g_{i j}\left(\partial_{t} N^{j}-N^{k} \partial_{k} N^{j}\right)-N^{-1} \partial_{i} N+{ }^{(3)} \Gamma_{i j k} g^{j k},
\end{gathered}
$$

where ${ }^{(3)} \Gamma_{i j k}$ is the Christoffel symbol associated with the three-metric $g_{i j}$. We see that $\Gamma_{\hat{t}}$ depends on the time derivative of the lapse, and that $\Gamma_{i}$ depends on the time derivative of the shift. It is natural then to impose slicing conditions using the $F_{\hat{t}}$ component of the target gauge source function, and to impose shift conditions through the spatial components $F_{i}$. Once $F_{\hat{t}}$ and $F_{i}$ are specified, the time component $F_{t}$ is obtained from the identity $F_{t}=$ $N F_{\hat{t}}+N^{k} F_{k}$. Finally, we will want to express the target gauge source function in terms of the first-order $\mathrm{GH}$ Einstein system variables $\left\{\psi_{a b}, \Pi_{a b}, \Phi_{i a b}\right\}$; therefore the expression for $\Gamma_{a}$ from Eq. (19) will be useful:

$$
\Gamma_{a}=g^{i j} \Phi_{i j a}+t^{b} \Pi_{b a}-\frac{1}{2} g_{a}{ }^{i} \psi^{b c} \Phi_{i b c}-\frac{1}{2} t_{a} \psi^{b c} \Pi_{b c} .
$$

The remainder of this section presents a list of target gauge source functions, $F_{a}$, that describe commonly used gauge conditions in numerical relativity. Slicing conditions are described in Sec. III A and shift conditions are given in Sec. III B. 


\section{A. Slicing conditions}

One of oldest gauge conditions used in numerical relativity is maximal slicing [6], where the trace of the extrinsic curvature of the $t=$ constant hypersurfaces vanishes: $K=0$. More generally constant curvature slicings are sometimes used, $K=K_{0}$, where $K_{0}$ is constant on each slice (but may be a specified function of time). This gauge condition can be written in the form $G_{\hat{t}}=0$, where

$$
G_{\hat{t}}=K_{0}-K=K_{0}-\frac{1}{2} g^{i j} \Pi_{i j}-g^{i j} t^{c} \Phi_{i j c} .
$$

Using Eq. (45) with Eqs. (48) and (49), we obtain

$$
\begin{aligned}
F_{\hat{t}}= & -\frac{1}{2} t^{a} t^{b} \Pi_{a b}-\rho_{1} K_{0}+\frac{1}{2}\left(\rho_{1}-1\right) g^{i j} \Pi_{i j} \\
& +\left(\rho_{1}-1\right) t^{a} g^{i j} \Phi_{i j a} .
\end{aligned}
$$

The choice of the arbitrary slicing gauge parameter, $\rho_{1}=$ 1 , gives a very simple expression for the constant curvature target gauge source function $F_{\hat{t}}$, but other choices may be more stable or more effective.

Perhaps the most widely used slicing conditions are various members of the family introduced by Bona and Massó [7]. These gauge conditions are evolution equations for the lapse $N$ having the general form

$$
\partial_{t} N-\sigma N^{k} \partial_{k} N=-N^{2} f(N)\left(K-K_{0}\right),
$$

where $f(N)$ is an arbitrary function of the lapse. The original gauge condition [7] uses $\sigma=1$. The particular case $f(N)=2 / N$ corresponds to the widely used one-plus$\log$ slicing condition [8-11]. An expression for the general form of these gauge conditions in terms of the first-order GH fields is given by

$$
\begin{aligned}
G_{\hat{t}}= & K_{0}-g^{i j} t^{a} \Phi_{i j a}-\frac{1}{2} g^{i j} \Pi_{i j}-\frac{1}{2 f(N)} t^{a} t^{b} \Pi_{a b} \\
& +\frac{1-\sigma}{2 N f(N)} N^{i} t^{a} t^{b} \Phi_{i a b} .
\end{aligned}
$$

Using this condition in Eq. (45) results in the needed target gauge source functions for these Bona-Massó slicing conditions:

$$
\begin{aligned}
F_{\hat{t}}= & \frac{\rho_{1}-f(N)}{2 f(N)} t^{a} t^{b} \Pi_{a b}-\frac{\rho_{1}(1-\sigma)}{2 N f(N)} N^{k} t^{a} t^{b} \Phi_{k a b} \\
& -\rho_{1} K_{0}+\left(\rho_{1}-1\right) t^{a} g^{i j} \Phi_{i j a}+\frac{1}{2}\left(\rho_{1}-1\right) g^{i j} \Pi_{i j} .
\end{aligned}
$$

The slicing gauge parameter choice $\rho_{1}=1$ makes this expression for the Bona-Massó gauge condition particularly simple; however, any choice with $\rho_{1} \neq 0$ is allowed.

\section{B. Shift conditions}

The simplest shift condition (from our perspective) is referred to as $\Gamma$ freezing [12]. This condition fixes the trace of the Christoffel symbol associated with the conformal spatial metric $\tilde{g}_{i j}=g^{\lambda} g_{i j}$, where $g \equiv \operatorname{det} g_{i j}$ and $\lambda$ is a constant. (Often $\lambda$ is chosen to be $\lambda=-\frac{1}{3}$ so that $\operatorname{det} \tilde{g}_{i j}=$ 1 , but any value is allowed.) The relevant trace of this conformal connection is defined by

$$
{ }^{(3)} \tilde{\Gamma}^{i} \equiv{ }^{(3)} \tilde{\Gamma}_{j k}^{i} \tilde{g}^{j k}=g^{-\lambda}\left(g^{i k} g^{j l}-\frac{1+\lambda}{2} g^{i j} g^{k l}\right) \Phi_{j k l} \text {. }
$$

The $\Gamma$-freezing shift condition simply requires that

$$
\partial_{t}{ }^{(3)} \tilde{\Gamma}^{i}=0 .
$$

For our purposes this must be translated into a condition on the spacetime metric and its first derivatives. This is accomplished by integrating Eq. (55) to obtain ${ }^{(3)} \tilde{\Gamma}^{i}=$ ${ }^{(3)} \tilde{\Gamma}^{i}(0)$, where ${ }^{(3)} \tilde{\Gamma}^{i}(0)$ is the trace evaluated at the initial time. This condition can be expressed in terms of firstorder GH fields as

$$
G_{i}=g^{\lambda} g_{i j}{ }^{(3)} \tilde{\Gamma}^{j}(0)-\left(\delta_{i}{ }^{l} g^{j k}-\frac{1+\lambda}{2} \delta_{i}^{j} g^{k l}\right) \Phi_{j k l} .
$$

Using Eqs. (45) and (48), this gauge condition is easily transformed into the needed target gauge source function:

$$
\begin{aligned}
F_{i}= & \frac{1}{2}\left[1-\rho_{2}(1+\lambda)\right] g^{j k} \Phi_{i j k}-\frac{1}{2} t^{a} t^{b} \Phi_{i a b}-t^{a} \Pi_{a i} \\
& -\rho_{2} g^{\lambda} g_{i j}^{(3)} \tilde{\Gamma}^{j}(0)+\left(\rho_{2}-1\right) g^{j k} \Phi_{j k i},
\end{aligned}
$$

where the shift gauge parameter $\rho_{2} \neq 0$ can be chosen freely. As a modest generalization we might also want to consider $\Gamma$-fixing conditions for which ${ }^{(3)} \tilde{\Gamma}^{i}$ is specified as a function of time. For example we might want to set ${ }^{(3)} \tilde{\Gamma}^{i}(t)={ }^{(3)} \tilde{\Gamma}^{i}(0) e^{-\mu t}$. This can be done by replacing ${ }^{(3)} \tilde{\Gamma}^{i}(0)$ with the desired ${ }^{(3)} \tilde{\Gamma}^{i}(t)$ in Eqs. (56) and (57).

The most commonly used shift conditions in the numerical relativity community are the $\Gamma$-driver conditions. The simplest of these can be written as the following evolution equations for the shift [10],

$$
\begin{gathered}
\partial_{t} N^{i}=B^{i}, \\
\partial_{t} B^{i}+\eta_{2} B^{i}=\nu \partial_{t}{ }^{(3)} \tilde{\Gamma}^{i},
\end{gathered}
$$

where ${ }^{(3)} \tilde{\Gamma}^{i}$ is the trace of the conformal spatial connection, Eq. (54), and $\nu$ and $\eta_{2}$ are adjustable constants. The parameter $\nu$ is usually set to $\nu=\frac{3}{4}$ on the basis of causality arguments $[9,10]$. But these arguments do not apply when the lapse and shift are evolved with the GH Einstein equations, so we leave $\nu$ as an adjustable parameter. Unfortunately this shift condition is not of the form $G_{i}=$ $G_{i}(x, \psi, \partial \psi)$, which is required by our gauge-driver system, because the right side of Eq. (59) depends on second derivatives of the spacetime metric. This particular $\Gamma$-driver condition, Eqs. (58) and (59), can be transformed however into the more useful form

$$
\partial_{t} N^{i}=\nu\left[{ }^{(3)} \tilde{\Gamma}^{i}-\eta_{2} \Upsilon^{i}\right],
$$




$$
\partial_{t} Y^{i}+\eta_{2} Y^{i}={ }^{(3)} \tilde{\Gamma}^{i}
$$

We note that Eqs. (60) and (61) are equivalent to Eqs. (58) and (59) when $\eta_{2} \neq 0$. This can be seen by differentiating $B^{i}=\nu\left[{ }^{(3)} \tilde{\Gamma}^{i}-\eta_{2} \Upsilon^{i}\right]$ with respect to time to determine that Eq. (59) is equivalent to Eq. (61).

This transformed $\Gamma$-driver condition does not depend on the second derivatives of the spacetime metric, so it is of the form required for our gauge-driver system. This $\Gamma$-driver condition, Eq. (60), can be written in terms of the first-order $\mathrm{GH}$ fields as

$$
\begin{aligned}
G_{i}= & -t^{a} \Pi_{a i}+\frac{1}{N} t^{a} N^{j} \Phi_{j a i}+\frac{\nu \eta_{2}}{N^{2}} g_{i j} \Upsilon^{j} \\
& -\frac{\nu}{N^{2} g^{\lambda}}\left(g_{i}{ }^{l} g^{j k}-\frac{1+\lambda}{2} g_{i}^{j} g^{k l}\right) \Phi_{j k l},
\end{aligned}
$$

where the auxiliary field $Y^{i}$ must be evolved using Eq. (61), and is treated as an independent dynamical field along with the $\mathrm{GH}$ and gauge-driver fields. The addition of Eq. (61) to the evolution system does not affect hyperbolicity. (The combined system has the additional characteristic fields $Y^{i}$, all of which have characteristic speed zero.) When evolving Eqs. (58) and (59), it is common practice to set $\partial_{t} N^{i}=0$ initially [10]; the equivalent condition in our notation is initially choosing $\eta_{2} \Upsilon^{i}={ }^{(3)} \tilde{\Gamma}^{i}$. The target gauge source function $F_{i}$ for this $\Gamma$ driver is obtained from Eq. (62) using Eqs. (45) and (48):

$$
\begin{aligned}
F_{i}= & \left(\frac{\nu \rho_{2}}{N^{2} g^{\lambda}}-1\right)\left(g^{j k} g_{i}{ }^{l}-\frac{1}{2} g_{i}{ }^{j} g^{k l}\right) \Phi_{j k l}-\frac{1}{2} t^{a} t^{b} \Phi_{i a b} \\
& -\frac{\rho_{2}}{N} t^{a} N^{j} \Phi_{j a i}-\frac{\nu \eta_{2} \rho_{2}}{N^{2}} g_{i j} \Upsilon^{j} \\
& -\frac{\nu \lambda \rho_{2}}{2 N^{2} g^{\lambda}} g^{j k} \Phi_{i j k}+\left(\rho_{2}-1\right) t^{a} \Pi_{a i} .
\end{aligned}
$$

The shift gauge parameter $\rho_{2}$ can be chosen to have any nonzero value.

\section{FLAT-SPACE STABILITY ANALYSIS}

The analysis of the gauge-driver equations in the previous sections is concerned with rather general questions, such as: Are the equations hyperbolic? What are the appropriate boundary conditions? How are particular gauge conditions implemented? In this section (and the next) we focus on questions about the stability and effectiveness of the gauge-driver equations, such as, Are the gauge-driver evolution equations stable? How well do the equations actually drive $H_{a}$ toward the target gauge source function $F_{a}$ ? In this section we use (mostly) analytical methods to explore these questions for simple cases that can be described as linear perturbations of flat spacetime. We consider three successively more complicated versions of this flat spacetime problem: First, we analyze the solutions to the gauge-driver equation with a fixed $F_{a}$ on a flat background spacetime. Second, we generalize this problem by allowing $F_{a}$ to have a prescribed time dependence. Third, we analyze the more realistic case of the coupled gaugedriver and GH Einstein systems for linear perturbations of flat spacetime. We present this analysis in some detail for the case of a target $F_{a}$ representing Bona-Massó slicing and a $\Gamma$-driver shift condition.

Before we specialize to these three specific problems however, we first establish some common notation and present the basic equations. Since we are perturbing about flat spacetime, it is convenient to decompose the solutions into spatial Fourier basis functions. Thus we assume that the spatial dependence of each of the perturbed fields is $e^{i k_{j} x^{j}}$, where $k_{j}$ is a (constant) wave vector, and $x^{j}$ are the spatial Cartesian coordinates. We assume that the gauge source function $H_{a}$, the target function $F_{a}$, and the timeaveraging field $\theta_{a}$ have the forms $H_{a}(t, x)=\delta H_{a}(t) e^{i k_{j} x^{j}}$, $F_{a}(t, x)=\delta F_{a}(t) e^{i k_{j} x^{j}}$, and $\theta_{a}(t, x)=\delta \theta_{a}(t) e^{i k_{j} x^{j}}$. We also assume that the spacetime metric $\psi_{a b}$ has the form $\psi_{a b}(t, x)=\eta_{a b}+\delta \psi_{a b}(t) e^{i k_{j} x^{j}}$, where $\eta_{a b}$ is the fixed background Minkowski metric with $N=1, g_{i j}=\delta_{i j}$, and $N^{i}$ constant. With these assumptions the linearized gauge-driver system, Eqs. (20)-(22) and (27), can be written in the form

$$
\partial_{t} \delta H_{a}-i \beta k \delta H_{a}=-\delta \Pi_{a}^{H}-\gamma_{1}^{H} N^{j}\left(\delta \Phi_{j a}^{H}-i k_{j} \delta H_{a}\right),
$$

$$
\begin{aligned}
\partial_{t} \delta \Pi_{a}^{H}+ & {\left[2 \mu_{2}\left(1-\xi_{2}\right)-i \beta k\right] \delta \Pi_{a}^{H} } \\
= & -i k^{j} \delta \Phi_{j a}^{H}+\eta_{1} \delta \theta_{a}+\mu_{1}^{2}\left(1-\xi_{1}\right)\left(\delta H_{a}-\delta F_{a}\right) \\
& -\gamma_{1}^{H} \gamma_{2}^{H} N^{j}\left(\delta \Phi_{j a}^{H}-i k_{j} \delta H_{a}\right), \\
\partial_{t}\left(\delta \Phi_{j a}^{H}-i k_{j} \delta H_{a}\right)= & i k_{j} \gamma_{1}^{H} N^{l}\left(\delta \Phi_{l a}^{H}-i k_{l} \delta H_{a}\right) \\
& -\left(\gamma_{2}^{H}-i \beta k\right)\left(\delta \Phi_{j a}^{H}-i k_{j} \delta H_{a}\right),
\end{aligned}
$$

$$
\begin{aligned}
\partial_{t} \delta \theta_{a}+\eta_{1} \delta \theta_{a}= & {\left[2 \mu_{2} i k \beta\left(1-\xi_{3}\right)+\mu_{1}^{2} \xi_{1}\right] \delta H_{a} } \\
& +\left[2 \mu_{2}\left(\xi_{3}-\xi_{2}\right)-i \beta k\right] \delta \Pi_{a}^{H}+i k^{j} \delta \Phi_{j a}^{H} \\
& -2 \mu_{2}\left(1-\xi_{3}\right) \gamma_{1}^{H} N^{j}\left(\delta \Phi_{j a}^{H}-i k_{j} \delta H_{a}\right) \\
& +\gamma_{1}^{H} \gamma_{2}^{H} N^{j}\left(\delta \Phi_{j a}^{H}-i k_{j} \delta H_{a}\right)-\mu_{1}^{2} \xi_{1} \delta F_{a},
\end{aligned}
$$

where $k^{2}=k^{j} k_{j}, \beta k=k_{j} N^{j}$, and contractions are done with the flat background metric.

This linearized gauge-driver system, Eqs. (64)-(67), can be simplified somewhat. We note that Eq. (66) implies that violations in the gauge constraint $\delta \mathcal{C}_{j a}^{H}=\delta \Phi_{j a}^{H}-i k_{j} \delta H_{a}$ always decrease toward zero exponentially on the time scale $1 / \gamma_{2}^{H}$. Since the system is linear, we can (without loss of generality) confine our attention to the constraint satisfying solutions, $\delta \Phi_{j a}^{H}=i k_{j} \delta H_{a}$. This condition and 
Eq. (64) can be used to eliminate the fields $\delta \Pi_{a}^{H}$ and $\delta \Phi_{j a}^{H}$ from the system, resulting in the following simplified evolution system for $\delta H_{a}$ and $\delta \theta_{a}$ :

$$
\begin{aligned}
& \partial_{t}^{2} \delta H_{a}+2\left[\mu_{2}\left(1-\xi_{2}\right)-i \beta k\right] \partial_{t} \delta H_{a} \\
&+\left[k^{2}\left(1-\beta^{2}\right)+\right.\left.\mu_{1}^{2}\left(1-\xi_{1}\right)-2 i \mu_{2} \beta k\left(1-\xi_{2}\right)\right] \delta H_{a} \\
&=-\eta_{1} \delta \theta_{a}+\mu_{1}^{2}\left(1-\xi_{1}\right) \delta F_{a}, \\
& \partial_{t} \delta \theta_{a}+\eta_{1} \delta \theta_{a}=-\left[2 \mu_{2}\left(\xi_{3}-\xi_{2}\right)-i \beta k\right] \partial_{t} \delta H_{a} \\
&-\left[k^{2}\left(1-\beta^{2}\right)-\mu_{1}^{2} \xi_{1}\right. \\
&\left.-2 i \mu_{2} \beta k\left(1-\xi_{2}\right)\right] \delta H_{a} \\
&-\mu_{1}^{2} \xi_{1} \delta F_{a} .
\end{aligned}
$$

We note that the linearized gauge-driver system, Eqs. (68) and (69), does not depend on the metric perturbations $\delta \psi_{a b}$, except through the target function $\delta F_{a}$. This rather weak coupling means that the gauge-driver equations just respond to whatever target $\delta F_{a}$ the gauge and spacetime geometry dictate. It makes sense then to investigate the intrinsic response of the gauge-driver system to a given $\delta F_{a}$. We consider two simple test cases. First, in Sec. IVA we consider the case where the target gauge source function, $\delta F_{a}$, is time independent. Second, in Sec. IV B we consider the more general case where $\delta F_{a}=$ $\delta F_{a}(t)$ is a prescribed function of time. Then finally, in Sec. IV C we consider the more interesting and realistic case where the gauge-driver and GH Einstein systems are coupled, using the target $\delta F_{a}$ appropriate for Bona-Massó slicing and a $\Gamma$-driver shift condition.

\section{A. Time-independent $\delta F_{a}$}

We consider first the case where the target gauge source function has the form $F_{a}=\delta F_{a} e^{i k_{j} x^{j}}$ for constant $\delta F_{a}$. We also assume that the shift of the background spacetime vanishes: $\beta=0$. In this case the general solution to Eqs. (68) and (69) has the form

$$
\begin{aligned}
& \delta H_{a}(t)=\delta F_{a}+\sum_{r} \delta H_{a}^{r} e^{s_{r} t}, \\
\delta \theta_{a}(t)=- & \frac{k^{2}}{\eta_{1}} \delta F_{a} \\
- & \sum_{r} \frac{k^{2}+2 s_{r} \mu_{2}\left(\xi_{3}-\xi_{2}\right)-\mu_{1}^{2} \xi_{1}}{s_{r}+\eta_{1}} \delta H_{a}^{r} e^{s_{r} t},
\end{aligned}
$$

where the $\delta H_{a}^{r}$ are constants and $s_{r}$ are the roots (assumed to be nondegenerate) of the characteristic polynomial,

$$
\begin{aligned}
0= & s_{r}^{3}+\left[2 \mu_{2}\left(1-\xi_{2}\right)+\eta_{1}\right] s_{r}^{2}+\left[k^{2}+\mu_{1}^{2}\left(1-\xi_{1}\right)\right. \\
& \left.+2 \mu_{2} \eta_{1}\left(1-\xi_{3}\right)\right] s_{r}+\eta_{1} \mu_{1}^{2} .
\end{aligned}
$$

Equation (72) is the necessary and sufficient condition that the solution satisfies Eqs. (68) and (69). The three roots of Eq. (72) consist of a real root, $s_{0}$, and a complex conjugate pair, $s_{ \pm}$.

Figure 1 illustrates the dependence of the real parts of the roots, $s_{0}$ and $s_{ \pm}$, on the wave number $k$ for the case $\mu=\mu_{1}=\mu_{2}=\eta_{1}$ and $0=\xi_{1}=\xi_{2}=\xi_{3}$. These roots have strictly negative real parts for all $k$, so the gauge source function $\delta H_{a}$ is always driven toward the target gauge source function $\delta F_{a}$. At least for this simple case, $\delta H_{a}$ approaches the target $\delta F_{a}$ exponentially.

Simple analytical expressions for the roots of the characteristic polynomial, Eq. (72), exist in the limits of small and large $k$. The large $k$ limit is the most interesting, because it describes the sufficiently short wavelength perturbations of any spacetime. The asymptotic expressions for the large $k$ roots are

$$
\begin{gathered}
\operatorname{Re}\left(s_{0}\right)=-\eta_{1}\left(\frac{\mu_{1}}{k}\right)^{2}+\mathcal{O}\left(k^{-4}\right), \\
\operatorname{Re}\left(s_{ \pm}\right)=-\mu_{2}\left(1-\xi_{2}\right)-\frac{\eta_{1}}{2}+\frac{\eta_{1}}{2}\left(\frac{\mu_{1}}{k}\right)^{2}+\mathcal{O}\left(k^{-4}\right) .
\end{gathered}
$$

These results show that the $s_{ \pm}$modes are damped at approximately the rate $\mu_{2}\left(1-\xi_{2}\right)+\eta_{1} / 2$ in the large $k$ limit, while the damping rate for the $s_{0}$ mode approaches zero. These modes are stable for large enough $k$, then, as long as $\eta_{1} \mu_{1}^{2}>0$ and $\mu_{2}\left(1-\xi_{2}\right)+\eta_{1} / 2>0$.

\section{B. Time-dependent $\delta F_{a}$}

Next we consider solutions to Eqs. (68) and (69) for the case where $\delta F_{a}$ is a specified function of time: $\delta F_{a}=$ $\delta F_{a}(t)$. In principle the equations could be solved analytically by Laplace transforming the equations in time, and solving for each frequency component of $\delta H_{a}(t)$ separately. Instead it is more straightforward, and perhaps more instructive, to integrate the equations numerically for some illustrative $\delta F_{a}(t)$. We assume for this simple example that the shift of the background spacetime van-

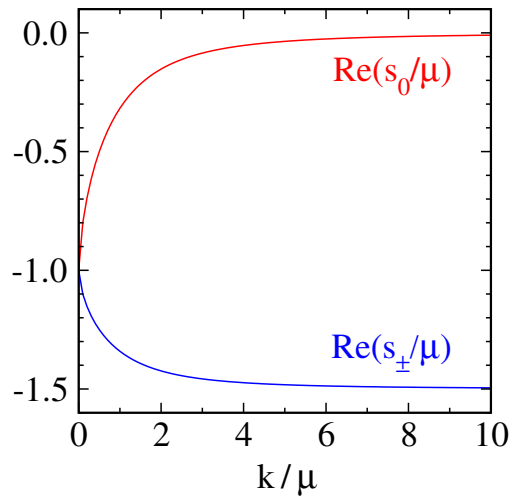

FIG. 1 (color online). Real part of the characteristic frequencies of the gauge-driver system: $s_{0}$ and $s_{ \pm}$. 
ishes, $\beta=0$, and the other parameters that determine the system take the values $\mu=\mu_{1}=\mu_{2}=\eta_{1}$ and $0=\xi_{1}=$ $\xi_{2}=\xi_{3}$. We have solved the resulting simplified equations numerically for the case $\delta F_{a}(t)=3+e^{-(t-10)^{2} / 9}$ with $k=$ 1. Equations (68) and (69) require initial conditions for $\delta H_{a}, \partial_{t} \delta H_{a}$, and $\delta \theta_{a}$. We use $\delta H_{a}(0)=\delta F_{a}(0)$, $\partial_{t} \delta H_{a}(0)=0$, and $\mu \delta \theta_{a}(0)=-k^{2} \delta H_{a}(0)$. These initial data for $\delta H_{a}$ and its time derivative were chosen to be fairly well matched with the target $\delta F_{a}$. They are similar to the initial conditions used in our more realistic tests in Sec. V. This target $\delta F_{a}$ changes significantly for times near $t=10$, so this test explores how well the gauge-driver system is able to track an evolving target $\delta F_{a}$. Figure 2 shows that the gauge-driver equation is fairly successful (at the few percent accuracy level) in driving $\delta H_{a}(t)$ toward $\delta F_{a}(t)$ for $\mu \gtrsim 2$ even in this rather dynamical situation.

\section{Coupled systems}

Finally we investigate the stability of the coupled gaugedriver and $\mathrm{GH}$ Einstein equations for perturbations of flat spacetime. The perturbed Einstein system reduces to a relatively simple form ${ }^{2}$ in this case:

$$
\eta^{c d} \partial_{c} \partial_{d} \delta \psi_{a b}+\partial_{a} \delta H_{b}+\partial_{b} \delta H_{a}=0 .
$$

We study the stability of the coupled system, Eqs. (68), (69), and (75), by Laplace transforming the equations in time, i.e., by considering solutions with time dependence $e^{\text {st }}$. In this case Eqs. (68) and (69) can be reduced to the single equation,

$$
P(s) \delta H_{a}=\mu_{1}^{2}\left(1-\frac{\xi_{1} s}{s+\eta_{1}}\right) \delta F_{a},
$$

where $P(s)$ is defined by

$$
\begin{aligned}
P(s)= & \hat{s}^{2}+2 \mu_{2}\left(1-\xi_{2}\right) \hat{s}+k^{2}+\mu_{1}^{2}\left(1-\xi_{1}\right) \\
& -\frac{\eta_{1}}{s+\eta_{1}}\left\{k^{2}-\mu_{1}^{2} \xi_{1}-2 i \beta k \mu_{2}\left(1-\xi_{3}\right)\right. \\
& \left.+\hat{s}\left[2 \mu_{2}\left(\xi_{3}-\xi_{2}\right)-i \beta k\right]\right\} .
\end{aligned}
$$

We use the notation $\hat{s} \equiv s-i k \beta$. The analogous expressions for the Laplace transform of the GH Einstein system, Eq. (75), are given by

$$
\begin{gathered}
0=\left(\hat{s}^{2}+k^{2}\right) \delta \psi_{\hat{t} \hat{t}}-2 \hat{s} \delta H_{\hat{t}}, \\
0=\left(\hat{s}^{2}+k^{2}\right) \delta \psi_{\hat{t} j}-\hat{s} \delta H_{j}-i k_{j} \delta H_{\hat{t}}, \\
0=\left(\hat{s}^{2}+k^{2}\right) \delta \psi_{j l}-i k_{j} \delta H_{l}-i k_{l} \delta H_{j},
\end{gathered}
$$

where $\delta H_{\hat{t}}=\delta H_{t}-N^{j} \delta H_{j}$, etc. These equations can be

\footnotetext{
${ }^{2}$ In this analysis we assume that the gauge constraint $\delta H_{a}+$ $\delta \Gamma_{a}=0$ is satisfied. The analysis of the GH Einstein constraint evolution system in Ref. [4] shows that violations of this constraint are damped exponentially for perturbations of flat spacetime.
}

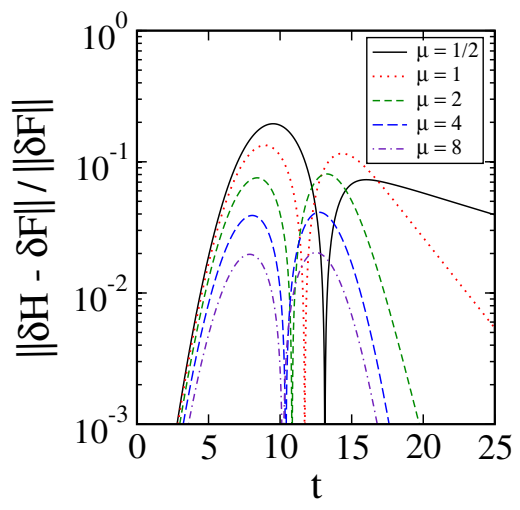

FIG. 2 (color online). Response of the gauge-driver system to a time-dependent $\delta F_{a}$ of the form $\delta F_{a}=3+e^{-(t-10)^{2} / 9}$, initial conditions $\delta H_{a}(0)=\delta F_{a}(0), \partial_{t} \delta H_{a}(0)=0$, and a range of values for the damping parameter $\mu=\mu_{1}=\mu_{2}=\eta_{1}$. This test uses $k=1$ and $\xi_{1}=\xi_{2}=\xi_{3}=0$.

used to express $\delta H_{a}$ and $\delta \psi_{j l}$ in terms of $\delta \psi_{\hat{t} a}$ for the case $\hat{s} \neq 0$ :

$$
\begin{gathered}
\delta H_{\hat{t}}=\frac{\hat{s}^{2}+k^{2}}{2 \hat{s}} \delta \psi_{\hat{t} \hat{t}}, \\
\delta H_{j}=\frac{\hat{s}^{2}+k^{2}}{\hat{s}^{2}}\left(\hat{s} \delta \psi_{\hat{t} j}-\frac{1}{2} i k_{j} \delta \psi_{\hat{t} \hat{t})},\right. \\
\delta \psi_{j l}=\hat{s}^{-2}\left(i \hat{s} k_{j} \delta \psi_{\hat{t} l}+i \hat{s} k_{l} \delta \psi_{\hat{t} j}+k_{j} k_{l} \delta \psi_{\hat{t} \hat{t}}\right) .
\end{gathered}
$$

The case $\hat{s}=0$ is essentially trivial: In this case $k^{2} \delta \psi_{\hat{t} \hat{t}}=$ $0, \quad k^{2} \psi_{\hat{t} j}=i k_{j} \delta H_{\hat{t}}, \quad k^{2} \delta \psi_{j l}=i k_{j} \delta H_{l}+i k_{l} \delta H_{j}, \quad$ and $\delta H_{a}=\delta F_{a}$. The metric perturbation in this case is pure gauge (an infinitesimal coordinate transformation generated by the time-independent $\delta H_{a} / k^{2}$ ), and the gauge source function $\delta H_{a}$ is identical to the target $\delta F_{a}$ in this case. So we focus on the $\hat{s} \neq 0$ case for the remainder of this discussion.

We consider in detail now the coupled gauge-driver system for the case of Bona-Massó slicing with $\sigma=0$, and the $\Gamma$-driver shift condition. The perturbed flat-space limit of $\delta F_{a}$ for the Bona-Massó driver, Eq. (53) with $\sigma=$ 0 , is given by

$$
\begin{aligned}
\delta F_{\hat{t}}= & {\left[\hat{s} \frac{f(1)-\rho_{1}}{2 f(1)}-\frac{i \beta k \rho_{1}}{2 f(1)}\right] \delta \psi_{\hat{t} \hat{t}}+i\left(\rho_{1}-1\right) k^{l} \delta \psi_{\hat{t} l} } \\
& -\frac{1}{2}\left(\rho_{1}-1\right) \hat{s} \delta \psi_{l}^{l},
\end{aligned}
$$

while the target for the $\Gamma$-driver shift condition, Eq. (63), reduces to

$$
\begin{aligned}
\delta F_{j}= & \left(\hat{s}-\rho_{2} s\right) \delta \psi_{\hat{t} j}+i\left(\frac{\nu \rho_{2} s}{s+\eta_{2}}-1\right) \delta \psi_{j l} k^{l}-\frac{i}{2} k_{j} \delta \psi_{\hat{t} \hat{t}} \\
& -\frac{i}{2}\left[\frac{\nu \rho_{2} s(1+\lambda)}{s+\eta_{2}}-1\right] k_{j} \delta \psi_{l}^{l} .
\end{aligned}
$$


The spatial metric perturbations, $\delta \psi_{j l}$, that appear in Eqs. (84) and (85) can be replaced by $\delta \psi_{\hat{t} a}$ using Eq. (83):

$$
\begin{aligned}
\delta F_{\hat{t}}=[\hat{s} & \left.\frac{f(1)-\rho_{1}}{2 f(1)}-\frac{i \beta k \rho_{1}}{2 f(1)}-\frac{k^{2}\left(\rho_{1}-1\right)}{2 \hat{s}}\right] \delta \psi_{\hat{t} \hat{t}} \\
\delta F_{j}= & \frac{i}{2}\left[\frac{k^{2}}{\hat{s}^{2}}\left(\nu \rho_{2} s \frac{1-\lambda}{s+\eta_{2}}-1\right)-1\right] k_{j} \delta \psi_{\hat{t} \hat{t}} \\
& -\left[\frac{k^{2}}{\hat{s}}\left(\frac{\nu \rho_{2} s}{s+\eta_{2}}-1\right)+\rho_{2} s-\hat{s}\right] \delta \psi_{\hat{t} j} \\
& +\frac{\nu \rho_{2} s \lambda}{\hat{s}\left(s+\eta_{2}\right)} k_{j} k^{l} \delta \psi_{\hat{t} l} .
\end{aligned}
$$

Now substitute these expressions for $\delta F_{a}$, Eqs. (86) and (87), and the expressions for $\delta H_{a}$, Eqs. (81) and (82), into the perturbed gauge-driver Eq. (76). The result is a system of linear algebraic equations for $\delta \psi_{\hat{t} a}$. This system can be decoupled, and nontrivial solutions exist if and only if the frequency $s$ satisfies one of the following characteristic polynomials:

$$
\begin{aligned}
0= & \frac{\hat{s}^{2}+k^{2}}{\hat{s}} P(s)+\mu_{1}^{2}\left(1-\frac{\xi_{1} s}{s+\eta_{1}}\right) \\
& \times\left[\hat{s} \frac{\rho_{1}-f(1)}{f(1)}+\frac{i \beta k \rho_{1}}{f(1)}+\left(\rho_{1}-1\right) \frac{k^{2}}{\hat{s}}\right], \\
0= & \frac{\hat{s}^{2}+k^{2}}{\hat{s}} P(s)+\mu_{1}^{2}\left(1-\frac{\xi_{1} s}{s+\eta_{1}}\right) \\
& \times\left[\frac{k^{2}}{\hat{s}}\left(\nu \rho_{2} s \frac{1-\lambda}{s+\eta_{2}}-1\right)+\rho_{2} s-\hat{s}\right], \\
0= & \frac{\hat{s}^{2}+k^{2}}{\hat{s}} P(s)+\mu_{1}^{2}\left[\frac{k^{2}}{\hat{s}}\left(\frac{\nu \rho_{2} s}{s+\eta_{2}}-1\right)+\rho_{2} s-\hat{s}\right],
\end{aligned}
$$

where $P(s)$ is defined in Eq. (77).

The flat-space stability analysis presented here is relevant to generic spacetimes when the wave number $k$ of the perturbation becomes sufficiently large. We have solved the characteristic polynomials in Eqs. (88)-(90) in this limit. The leading order expressions for the real parts of these roots are given as follows. For the time slicing modes (in which $\delta \psi_{\hat{t} \hat{t}} \neq 0$ ), the roots of Eq. (88), we have

$$
\begin{aligned}
& \operatorname{Re}(s)=-\frac{\eta_{1} \mu_{1}^{2} \rho_{1}}{\left(1-\beta^{2}\right)^{2} k^{2}}+\mathcal{O}\left(k^{-4}\right), \\
\operatorname{Re}(s)= & \pm \frac{1}{4}\left\{\left[\eta_{1}+2 \mu_{2}\left(1-\xi_{2}\right)\right]^{2}\right. \\
& \left.-4 \mu_{1}^{2} \rho_{1}\left(1-\xi_{1}\right) \frac{1-f(1) \pm \beta}{f(1)}\right\}^{1 / 2} \\
& -\frac{1}{4}\left[\eta_{1}+2 \mu_{2}\left(1-\xi_{2}\right)\right]+\mathcal{O}\left(k^{-2}\right) .
\end{aligned}
$$

The asymptotic forms of the roots of the longitudinal modes (in which $k^{j} \delta \psi_{\hat{t} j} \neq 0$ ), Eq. (89), are given by

$$
\operatorname{Re}(s)=-\eta_{2}+\mathcal{O}\left(k^{-2}\right),
$$

$$
\begin{aligned}
\operatorname{Re}(s)= & \pm \frac{1}{4}\left\{\left[\eta_{1}+2 \mu_{2}\left(1-\xi_{2}\right)\right]^{2}\right. \\
& \left.-4 \mu_{1}^{2} \rho_{2}\left(1-\xi_{1}\right)[1 \pm \beta-\nu(1-\lambda)]\right\}^{1 / 2} \\
& -\frac{1}{4}\left[\eta_{1}+2 \mu_{2}\left(1-\xi_{2}\right)\right]+\mathcal{O}\left(k^{-2}\right) .
\end{aligned}
$$

Finally the asymptotic forms of the roots of the transverse modes (in which $\left[k^{2} g^{i j}-k^{i} k^{j}\right] \delta \psi_{\hat{t} j} \neq 0$ ), Eq. (90), are given by

$$
\begin{aligned}
& \operatorname{Re}(s)=-\eta_{2}+\mathcal{O}\left(k^{-2}\right), \\
\operatorname{Re}(s)= & \pm \frac{1}{4}\left\{\left[\eta_{1}+2 \mu_{2}\left(1-\xi_{2}\right)\right]^{2}\right. \\
& \left.-4 \mu_{1}^{2} \rho_{2}\left(1-\xi_{1}\right)[1 \pm \beta-\nu]\right\}^{1 / 2} \\
& -\frac{1}{4}\left[\eta_{1}+2 \mu_{2}\left(1-\xi_{2}\right)\right]+\mathcal{O}\left(k^{-2}\right) .
\end{aligned}
$$

All four \pm sign combinations represent distinct roots in Eqs. (92), (94), and (96). Stability of the gauge-driver system requires $\operatorname{Re}(s)<0$. Therefore, stability of the short wavelength modes requires the following inequalities on the system parameters:

$$
\begin{gathered}
0<\eta_{1} \rho_{1}, \\
0<\eta_{1}+2 \mu_{2}\left(1-\xi_{2}\right), \\
0<\rho_{1}\left(1-\xi_{1}\right) \frac{1-f(1) \pm \beta}{f(1)}, \\
0<\eta_{2}, \\
0<\rho_{2}\left(1-\xi_{1}\right)[1 \pm \beta-\nu(1-\lambda)], \\
0<\rho_{2}\left(1-\xi_{1}\right)[1 \pm \beta-\nu] .
\end{gathered}
$$

We note that these conditions can be satisfied for small values of $\beta$ by taking $\eta_{1}>0, \eta_{2}>0, \mu_{2}>0, \xi_{1}<1$, $\xi_{2}<1, \rho_{1}>0, \rho_{2}>0,0<f(1)<1, \nu<1, \nu(1-\lambda)<1$.

We have also explored the roots of these characteristic polynomials numerically. Figure 3 illustrates $\max [\operatorname{Re}(s)]$, the root of these equations having the largest real part, as a function of the parameter $f(1)$ that characterizes the BonaMassó slicing condition in this flat-space limit. The curves correspond to the roots for the driver system with various values of $\mu=\mu_{1}=\mu_{2}=\eta_{1}=\frac{1}{32} \eta_{2}, \lambda=-\frac{1}{3}, \nu=\frac{3}{4}$, and $k=1$. These parameter values were chosen because they satisfy the inequalities in Eqs. (97)-(102), and because they perform fairly well for the 3D numerical tests discussed in Sec. V. We see that the maximum real part of $s$ is negative for $f(1)$ in the range $0<f(1)<1$, and so the coupled gauge-driver system is stable for these values. The system is most stable for $f(1) \approx \frac{1}{2}$, so we adopt this value 


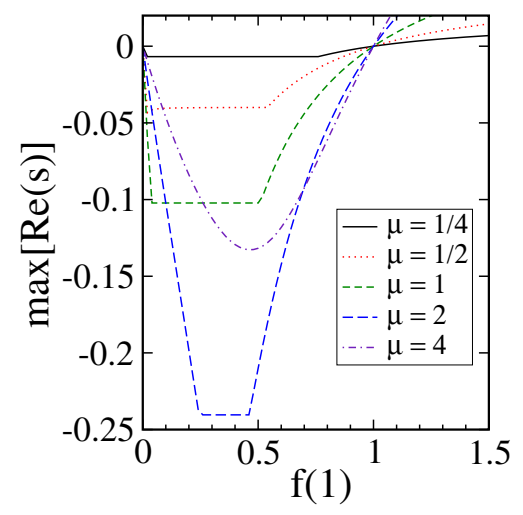

FIG. 3 (color online). Maximum damping rate of the modes as a function of the Bona-Massó slicing condition parameter $f(1)$. The other parameters used for this case are $k=1, \beta=0, \mu=$ $\mu_{1}=\mu_{2}=\eta_{1}=\frac{1}{32} \eta_{2}, \xi_{1}=\xi_{2}=\xi_{3}=0, \rho_{1}=\rho_{2}=\frac{1}{2}, \nu=$ $\frac{3}{4}$, and $\lambda=-\frac{1}{3}$.

in our numerical tests of the gauge-driver system in Sec. V. We also note that the standard value, $f(1)=2$, used for one-plus-log slicing by most of the numerical relativity community [8-11] is unstable when used in our gaugedriver equations. This does not imply that $f(1)=2$ is a bad choice when used in a standard three-plus-one evolution, only that it is unstable when used with our gauge-driver system.

Figure 4 illustrates the $k$ dependence of $\max [\operatorname{Re}(s)]$ for a range of values of the gauge-driver damping coefficients $\mu=\mu_{1}=\mu_{2}=\eta_{1}=\frac{1}{32} \eta_{2}$. For short wavelength perturbations, i.e., for values of $k$ with $k \gtrsim \mu, \max [\operatorname{Re}(s)]$ decreases as $\mu$ increases. Thus the solutions with large $k$ are damped more effectively as $\mu$ increases. However, for long wavelength perturbations, i.e., for values of $k$ with $k \lesssim \mu$, $\max [\operatorname{Re}(s)]$ increases as $\mu$ increases. Thus the solutions with small $k$ are less efficiently damped as $\mu$ increases. It follows that there is an optimal value of $\mu$ to use for any particular problem: choose $\mu \approx k_{c}$, where $1 / k_{c}$ corre-

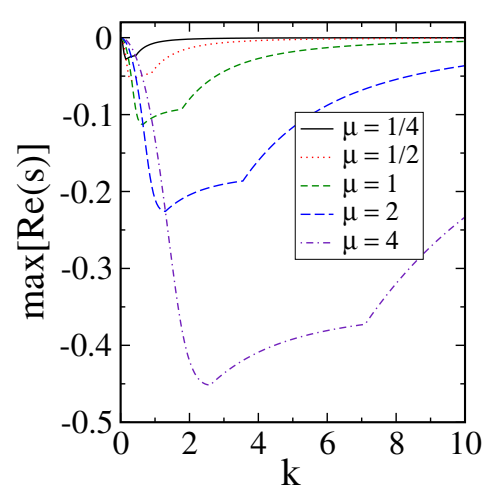

FIG. 4 (color online). Maximum damping rate of the modes as a function of the wave number $k$. The other parameters used for this case are $\beta=0, \mu=\mu_{1}=\mu_{2}=\eta_{1}=\frac{1}{32} \eta_{2}, \xi_{1}=\xi_{2}=$ $\xi_{3}=0, \rho_{1}=\rho_{2}=\frac{1}{2}, f(1)=\frac{1}{2}, \nu=\frac{3}{4}$, and $\lambda=-\frac{1}{3}$.

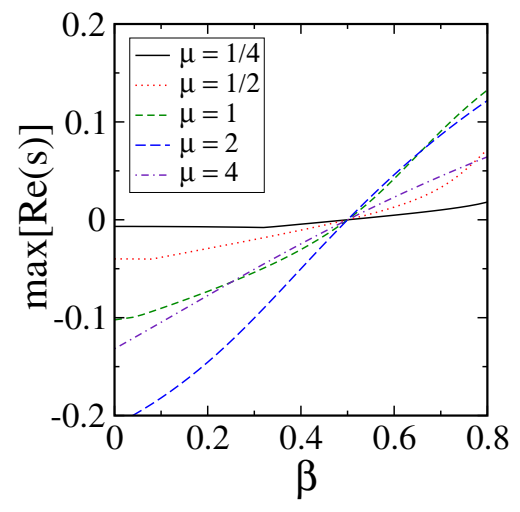

FIG. 5 (color online). Maximum damping rate of the modes as a function of the shift parameter $\beta$. The other parameters used for this case are $k=1, \mu=\mu_{1}=\mu_{2}=\eta_{1}=\frac{1}{32} \eta_{2}, \xi_{1}=$ $\xi_{2}=\xi_{3}=0, \rho_{1}=\rho_{2}=\frac{1}{2}, f(1)=\frac{1}{2}, \nu=\frac{3}{4}$, and $\lambda=-\frac{1}{3}$.

sponds to the length scale on which the gauge condition needs to be enforced most effectively.

Figure 5 illustrates the dependence of $\max [\operatorname{Re}(s)]$ on the background shift parameter $\beta$ for a range of values of the gauge-driver damping coefficients $\mu=\mu_{1}=\mu_{2}=\eta_{1}=$ $\frac{1}{32} \eta_{2}$. For small values of $\beta$ we see that the system is stable; however, for $\beta>\frac{1}{2}$ the system becomes unstable. This instability may be important in more realistic problems that involve black holes. Even for single black-hole spacetimes, the usual time-independent coordinate representations have nonvanishing shifts with $\beta \approx 1$ near the horizon. Binary black-hole spacetimes also use large shifts (with $\beta>1$ in many cases) when coordinates that corotate with the black holes are used. We explore the stability of this gauge-driver system for the case of single black-hole spacetimes in Sec. V.

We have also examined several other slicing and shift conditions using these perturbation techniques. As a consequence of Sec. IVA, our gauge-driver system is stable for harmonic slicing $\delta F_{\hat{t}}=0$ and harmonic shift $\delta F_{i}=0$. We also find that the combinations of a stable Bona-Massó slicing condition with harmonic shift, and of harmonic slicing with the $\Gamma$-driver shift condition, are stable. However, we find that the maximal slicing and $\Gamma$-freezing conditions are unconditionally unstable when enforced through our gauge-driver equations.

\section{NUMERICAL TESTS}

In this section we describe the results of 3D numerical tests of the gauge-driver system. We consider two cases: first a Schwarzschild black hole with perturbed lapse and shift, and second a Schwarzschild black hole with a superimposed outgoing physical gravitational wave pulse. The full coupled nonlinear GH Einstein and gauge-driver systems are solved numerically for these cases. We measure the stability and effectiveness of the gauge-driver system in 
these tests as it attempts to drive the gauge toward BonaMassó slicing and $\Gamma$-driver shift conditions.

These numerical tests are conducted using the infrastructure of the Caltech/Cornell Spectral Einstein Code (SpEC). This code uses pseudospectral collocation methods, as described, for example, in Refs. [13,14]. We use the generalized harmonic form of the Einstein equations, as described in Ref. [4]. The evolution equations for the combined GH Einstein and the gauge-driver system are integrated in time using the adaptive fifth-order Cash-Karp method [15]. We use a form of spectral filtering, as described in Ref. [13], that sets to zero in each time step the changes in the top four tensor spherical harmonic expansion coefficients of each of our evolved quantities. This filtering step is needed to eliminate an instability associated with the inconsistent mixing of tensor spherical harmonics in our approach.

Initial conditions are needed for any evolution of the combined GH Einstein and gauge-driver systems, and these initial data consist of the spacetime metric $\psi_{a b}$, the gauge source function $H_{a}$, and their time derivatives. For the tests described here we take the initial spacetime metric $\psi_{a b}$ to be the Schwarzschild geometry plus perturbations as described in Secs. VA and V B. We set the time derivatives of the spatial components of the metric to zero for the pure gauge perturbation test in Sec. VA, and equal to the appropriate time derivative of the superimposed physical gravitational wave pulse for the test in Sec. VB. The remaining initial data needed for these evolutions, $\partial_{t} N$, $\partial_{t} N^{i}, H_{a}$, and $\partial_{t} H_{a}$, are pure gauge quantities. The time derivatives of the lapse and shift are chosen here to ensure that $H_{a}$ satisfies the desired gauge condition, $H_{a}=F_{a}$, initially. And finally the initial value of $H_{a}$ is chosen here to ensure that the gauge constraint, $\mathcal{C}_{a}=H_{a}+\Gamma_{a}=0$, vanishes initially.

\section{A. Black hole with gauge perturbation}

For this test we consider a Schwarzschild black hole with perturbations in the lapse and shift. For the unperturbed hole we use isotropic spatial coordinates and maximal time slices $[16,17]$. The unperturbed spatial metric in this representation is given by

$$
d s^{2}=g_{i j} d x^{i} d x^{j}=\left(\frac{R}{r}\right)^{2}\left(d x^{2}+d y^{2}+d z^{2}\right),
$$

where $r^{2}=x^{2}+y^{2}+z^{2}$, and $R(r)$ (the areal radius) satisfies the differential equation,

$$
\frac{\mathrm{d} R}{\mathrm{~d} r}=\frac{R}{r} \sqrt{1-\frac{2 M}{R}+\frac{C^{2}}{R^{4}}}
$$

The constant $M$ is the mass of the hole, and $C$ is a parameter that specifies the particular maximal slicing. Finally, the unperturbed lapse $N$ and shift $N^{i}$ for this representation of Schwarzschild are given by

$$
\begin{gathered}
N=\sqrt{1-\frac{2 M}{R}+\frac{C^{2}}{R^{4}}}, \\
N^{i}=\frac{C \hat{r}^{i}}{R^{2}}\left(1-\frac{2 M}{R}+\frac{C^{2}}{R^{4}}\right),
\end{gathered}
$$

where $\hat{r}^{i}$ is the outward directed radial unit vector: $g_{i j} \hat{r}^{i} \hat{r}^{j}=1$.

We perturb this spacetime by changing the initial values of the lapse and shift, and their time derivatives. This type of perturbation changes the spacetime coordinates (or gauge) of the solution, but not its geometry. For these tests we perturb the lapse and shift of Eqs. (105) and (106) by adding

$$
\begin{array}{r}
\delta N=A \sin \left(2 \pi r / r_{0}\right) e^{-\left(r-r_{c}\right)^{2} / w^{2}} Y_{l m}, \\
\delta N^{i}=A \sin \left(2 \pi r / r_{0}\right) e^{-\left(r-r_{c}\right)^{2} / w^{2}} Y_{l m} \hat{r}^{i},
\end{array}
$$

where $Y_{l m}$ is the standard scalar spherical harmonic. In our numerical tests we use the background metric with $C=$ $1.73 M^{2}$, and perturbations with $A=0.01, r_{c}=15 M, w=$ $3 M, l=2, m=0$, and various values of the radial wavelength $r_{0}$.

We perform these numerical tests on a computational domain consisting of a spherical shell that extends from $r=0.78 M$ (just inside the horizon in these coordinates) to $r=30 M$. We divide this domain into eight subdomains. In each subdomain we express each Cartesian component of each dynamical field as a sum of Chebyshev polynomials of $r$ (through order $N_{r}-1$ ) multiplied by scalar spherical harmonics (through order $L$ ). The radii of the inner and outer edges of the various subdomains are adjusted to distribute the truncation error for this problem more or less uniformly. The specific radii of the subdomain boundaries used in this test are $0.78 M, 2.38 M, 4.6 M$, $8.83 M, 13.07 M, 17.30 M, 21.53 M, 25.77 M$, and $30.0 M$, respectively. The values of the parameters associated with the gauge-driver system used for this test are $\nu=\frac{3}{4}, \sigma=$ $0, \lambda=-\frac{1}{3}, \rho_{1}=\rho_{2}=\frac{1}{2}, \xi_{1}=\xi_{2}=\xi_{3}=0$, and various values of the parameter $\mu=\mu_{1}=\mu_{2}=\eta_{1}=\frac{1}{32} \eta_{2}$. The Bona-Massó slicing condition used here has $f(N)=$ $1 / 2 N$, and includes a target value for the extrinsic curvature $K_{0}$; for this test we set $K_{0}=0$.

Figure 6 illustrates the constraint violations for a set of representative evolutions from this test, and demonstrates the exponential convergence of our numerical method. The solid curves represent the constraints associated with the GH Einstein system, while the dotted curves represent the constraints of the gauge-driver system. We measure the constraint violations of the GH Einstein system for these tests using the norm $\left\|\mathcal{C}_{\mathrm{GH}}\right\|$ defined in Eq. (71) of Ref. [4]. The norm $\left\|\mathcal{C}_{\mathrm{GH}}\right\|$ is scaled so that it becomes of order unity when constraint violations start to dominante the solution. We define an analogous norm $\left\|\mathcal{C}_{\mathrm{H}}\right\|$ for the gauge-driver 


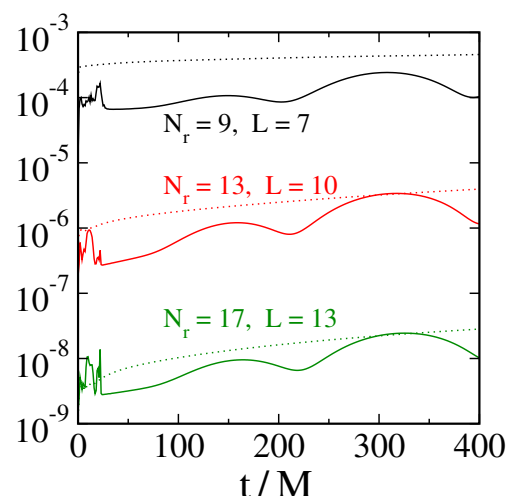

FIG. 6 (color online). Solid curves show the constraints of the $\mathrm{GH}$ Einstein system $\left\|\mathcal{C}_{\mathrm{GH}}\right\|$, dotted curves show the constraints of the gauge-driver system $\left\|\mathcal{C}_{\mathrm{H}}\right\|$ for a test with $\mu=1.0 / M$ and radial wavelength $r_{0}=6.0 \mathrm{M}$ evolved at several numerical resolutions.

system:

$$
\begin{aligned}
\left\|\mathcal{C}_{\mathrm{H}}\right\|^{2}= & \int \sqrt{g} m^{a b} g^{i j}\left(\mathcal{C}_{i a}^{H} \mathcal{C}_{j b}^{H}+g^{k l} \mathcal{C}_{i k a}^{H} \mathcal{C}_{j l b}^{H}\right) d^{3} x \\
& \times\left[\int \sqrt { g } m ^ { c d } g ^ { i j } \left(\partial_{i} H_{c} \partial_{j} H_{d}+\partial_{i} \Pi_{c}^{H} \partial_{j} \Pi_{d}^{H}\right.\right. \\
& \left.\left.+g^{k l} \partial_{i} \Phi_{k c}^{H} \partial_{j} \Phi_{l d}^{H}\right) d^{3} x\right]^{-1} .
\end{aligned}
$$

The quantity $m^{a b}$ is a positive definite matrix, which we set to the identity matrix, $m^{a b}=\delta^{a b}$, for these tests. Figure 6 shows the constraints for a particular test run with $\mu=$ $1.0 / M$ and $r_{0}=6.0 M$. The analogous graphs for the other tests reported here are qualitatively similar, with somewhat larger but still convergent "spikes" in $\left\|\mathcal{C}_{\mathrm{G} H}\right\|$ at early times $(t \lesssim 25 M)$ for the $\mu=0.5 / M$ case. Figure 6 shows that the constraints are well satisfied in our evolutions, and demonstrates that our numerical methods are (exponentially) convergent. The growth in the constraints seen at late times is a mild power law, approximately $t^{3 / 4}$, and is not something that concerns us.

Figure 7 illustrates the effectiveness of the gauge-driver system, at least for this test problem. We measure the difference between the gauge source function $H_{a}$ and the target function to which it is being driven, $F_{a}$, using the following $L^{2}$ norm:

$$
\frac{\|H-F\|^{2}}{\|F\|^{2}}=\frac{\int \sqrt{g} m^{a b}\left(H_{a}-F_{a}\right)\left(H_{b}-F_{b}\right) d^{3} x}{\int \sqrt{g} m^{c d} F_{c} F_{d} d^{3} x},
$$

where (as before) the matrix $m^{a b}$ is set to the identity, $m^{a b}=\delta^{a b}$, for these tests. This norm is scaled so that $H_{a}$ bears little resemblance to the target $F_{a}$ whenever the norm becomes of order unity. Figure 7 shows that the gauge perturbation used in this test violates the desired gauge conditions rather severely at early times. The norm $\|H-F\| /\|F\|$ is driven to values as large as 0.7 at about $t=20 M$ when the ingoing part of the gauge perturbation

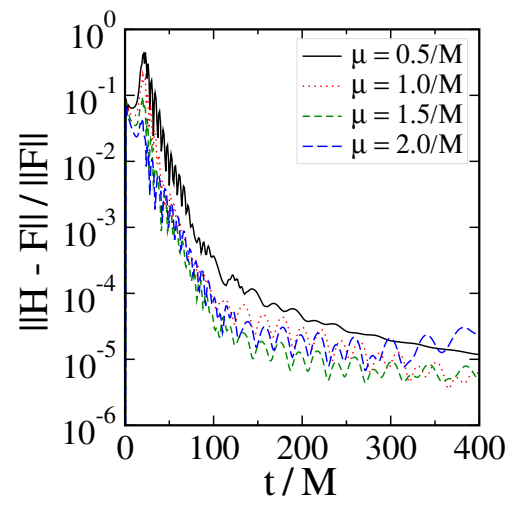

FIG. 7 (color online). Effectiveness of the gauge-driver equation is demonstrated by showing $\|H-F\| /\|F\|$ for evolutions with radial wavelength $r_{0}=6 M$ and several values of the gauge damping parameter $\mu M \in\left\{\frac{1}{2}, 1, \frac{3}{2}, 2\right\}$. These tests evolve a Schwarzschild black hole with strongly perturbed lapse and shift.

interacts most strongly with the black hole. After this initial interaction, the gauge-driver system takes over and effectively drives $\|H-F\| /\|F\|$ to values below $10^{-3}$ on time scales of $40 M$ to $60 M$, depending on the value of the gauge damping parameter $\mu$ used in the evolution. Figure 7 shows evolutions of gauge perturbations with radial wavelength $r_{0}=6 M$, and several values of the damping parameter $\mu M \in\left\{\frac{1}{2}, 1, \frac{3}{2}, 2\right\}$ computed with numerical resolution $N_{r}=17$ and $L=13$. The gauge-driver system is more effective at reducing $\|H-F\| /\|F\|$ quickly at early times, $t<75 M$, for larger values of $\mu$.

The $\mu=2 / M$ case shown in Fig. 7 has a mild instability that first appears at about $t=300 M$. This is a gauge instability, since it does not affect any of the constraint quantities. Also, for the runs we have done this instability appears convergent, in the sense that $\|H-F\| /\|F\|$ decreases as the numerical resolution is increased. Signs of this instability can be seen at some level for every value of $\mu$ for the numerical resolutions used here, starting at about $t=4000 M$ for $\mu=0.5 / M$, at about $t=600 M$ for $\mu=$ $1.0 / M$, and at about $t=450 M$ for $\mu=1.5 / M$. Larger values of $\mu$ are progressively more unstable. We do not understand the root cause(s) of this instability, but it seems likely that it is related to our rather simplistic boundary conditions, or the inherent instability of our coupled system when large shifts are present. Since the time scale of this instability is much longer than the time scale on which $H_{a}$ is driven toward $F_{a}$ (the effect we were interested in demonstrating here), we have not put a great deal of effort into completely understanding it for this simple test problem. Figure 8 provides some additional insight into the way the gauge-driver equation responds to different perturbations. The evolutions shown in Fig. 8 are all performed with $\mu=0.5 / M$ but several different values of the radial wavelength of the gauge perturbation: $r_{0} \in$ $\{4 M, 6 M, 8 M, 10 M\}$. These tests show that the gaugedriver system causes $\|H-F\| /\|F\|$ to approach zero 


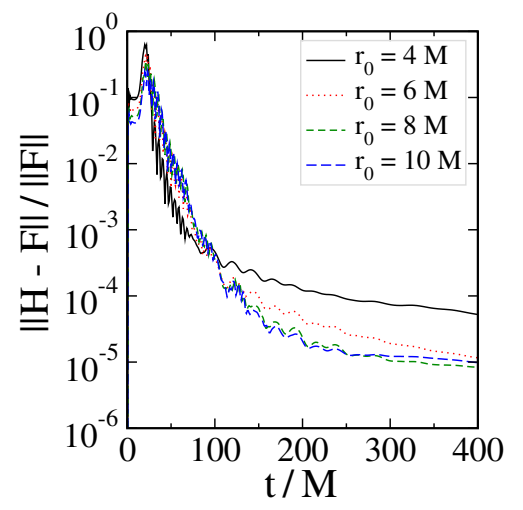

FIG. 8 (color online). Effectiveness of the gauge-driver equation is demonstrated by showing $\|H-F\| /\|F\|$ for evolutions with $\mu=0.5 / M$ and several values of the radial wavelength of the perturbation $r_{0} \in\{4 M, 6 M, 8 M, 10 M\}$. These tests evolve a Schwarzschild black hole with strongly perturbed lapse and shift.

more quickly (at least at early times) for shorter wavelength perturbations. Our hope is that this ability to efficiently control short wavelength features of the gauge is what will be needed to prevent the kinds of localized gauge singularities that often appear in our evolutions of binary black-hole spacetimes.

\section{B. Black hole with physical perturbation}

Our second numerical test of the gauge-driver system uses a Schwarzschild black hole with a superimposed outgoing gravitational wave pulse, as described in Refs. $[13,18]$. The background solution is a Schwarzschild black hole in Kerr-Schild coordinates,

$$
d s^{2}=-d t^{2}+\frac{2 M}{r}(d t+d r)^{2}+d x^{2}+d y^{2}+d z^{2},
$$

where $r^{2}=x^{2}+y^{2}+z^{2}$ and $M$ is the mass. We superimpose an odd-parity outgoing quadrupolar gravitational wave perturbation constructed using Teukolsky's method [19]. Its generating function is taken to be a Gaussian, $G(r)=A \exp \left[-\left(r-r_{c}\right)^{2} / w^{2}\right]$, with $A=4 \times 10^{-3}, r_{c}=$ $5 M$, and $w=1.5 M$. Using this perturbed Schwarzschild solution as the input conformal metric, the full nonlinear initial value equations (in the conformal thin sandwich formulation) are solved to obtain initial data that satisfy the constraints [20]. This procedure yields initial values for the spatial metric, extrinsic curvature, lapse, and shift. We note that the resulting solution to the constraints is still nearly (but not completely) outgoing.

The computational domain for this test problem is taken to be a spherical shell extending from $r=1.9 M$ (just inside the horizon in these coordinates) out to $r=$ 41.9M. This domain is subdivided into four spherical-shell subdomains of width $\Delta r=10 \mathrm{M}$. On each subdomain, the numerical solution is expanded in Chebyshev polynomials and spherical harmonics as before. For these tests we use numerical resolutions with $N_{r} \in\{21,31,41,51\}$ coeffi-

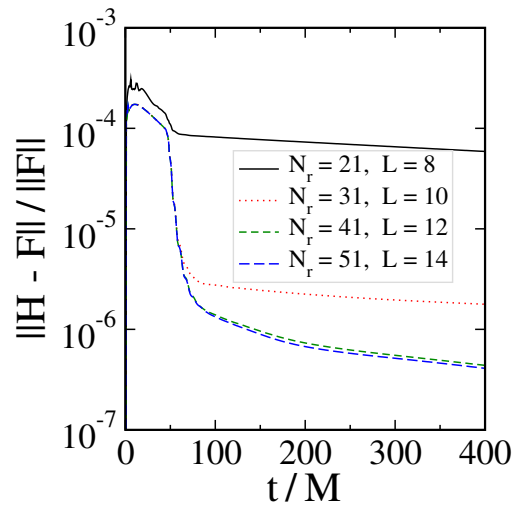

FIG. 9 (color online). Effectiveness of the gauge-driver equation is demonstrated by showing $\|H-F\| /\|F\|$ for evolutions with $\mu=0.25 / M$ obtained with a variety of numerical resolutions. This test uses a Schwarzschild black hole with a superimposed outgoing gravitational wave pulse.

cients per subdomain for the Chebyshev series and $l \leq L$ with $L \in\{8,10,12,14\}$ for the spherical harmonics. The values of the parameters associated with the gauge-driver system used for this test are $\nu=\frac{3}{4}, \sigma=0, \lambda=-\frac{1}{3}, \rho_{1}=$ $\rho_{2}=\frac{1}{2}, \xi_{1}=\xi_{2}=\xi_{3}=0$, and various values of the parameter $\mu=\mu_{1}=\mu_{2}=\eta_{1}=\frac{1}{32} \eta_{2}$. The Bona-Massó slicing condition used here has $f(N)=1 / 2 N$, and includes a target value for the extrinsic curvature $K_{0}$ that is set equal to its value in the perturbed initial data.

Figure 9 illustrates the effectiveness of the gauge-driver equation for imposing the Bona-Massó slicing and $\Gamma$-driver shift conditions in evolutions of a Schwarzschild black hole with physical gravitational wave perturbation. These tests were performed with the gauge damping parameter $\mu=0.25 / M$. For this test we set the target value for the extrinsic curvature $K_{0}$ to that of an unperturbed Kerr-Schild spacetime. The various curves in Fig. 9 illustrate how $\|H-F\| /\|F\|$ changes for evolutions performed with different numerical resolutions. The results are qualitatively similar to those of the first test: the black hole with physical gravitational wave perturbation does not satisfy the target gauge conditions exactly at early times, but the gauge-driver equation reduces $\|H-F\| /\|F\|$ to very small values by about $t=75 \mathrm{M}$. This test is less severe in some sense than our first pure gauge perturbation test, since the initial data in this case contains an outgoing gravitational wave pulse that never interacts very strongly with the black hole.

\section{DISCUSSION}

We have presented a new gauge-driver evolution system in Sec. II that makes it possible to impose a wide range of gauge conditions in the GH formulation of the Einstein equations, without destroying its hyperbolicity. The key idea is to construct an auxiliary hyperbolic evolution equation for the gauge source function $H_{a}$ that drives it toward 
the desired target $F_{a}$. Section III shows how many of the gauge conditions widely used by the numerical relativity community can be included in this way. In Sec. IV we analyze the effectiveness and stability of the combined GH Einstein and gauge-driver system for the case of perturbations of flat spacetime. This analysis shows that the gaugedriver equation effectively drives $H_{a}$ toward $F_{a}$, when $F_{a}$ is specified a priori as a function of the spacetime coordinates. We were somewhat surprised to find, however, that the gauge-driver system can be quite unstable when it is coupled to the GH Einstein system. We found that common gauge conditions like maximal slicing and the $\Gamma$-freezing gauge conditions are unconditionally unstable when implemented using our gauge-driver equation. This does not imply of course that those conditions are unsuitable for use with other forms of the Einstein system (like the Baumgarte-Shapiro-Shibata-Nakamura, BSSN, formulation [12]), just that they cannot be implemented in a completely stable way in the GH Einstein system coupled to the particular gauge-driver equations introduced here. Fortunately, we were able to find some of the commonly used gauge conditions that can be implemented in this way: certain Bona-Massó slicing conditions and a commonly used form of the $\Gamma$-driver shift conditions. Our 3D numerical tests in Sec. V show that the gauge-driver system can impose these gauge conditions stably and effectively for the evolutions of perturbed single black-hole spacetimes.

There has been a great deal of discussion in the literature about the formation of shocks when certain dynamical gauge conditions are imposed [21-23]. However, these discussions do not apply when those same gauge conditions are imposed using a driver condition. The gaugedriver system imposes the desired gauge condition only approximately, not exactly. At best, the desired gauge condition is imposed exactly only asymptotically in time as the system approaches a time-independent equilibrium state, and even in this state shocks do not necessarily form. On the contrary, there are many solutions even to bad gauge conditions that do not have shocks. What determines whether an evolution system develops shocks is the structure of the operator that evolves the spacetime metric and auxiliary fields. Our evolution system (including the gauge-driver system) has been carefully designed to be linearly degenerate, a condition that prevents the formation of shocks (resulting from a crossing of characteristics) from smooth initial data [24]. Linear degeneracy does not prevent the formation of curvature singularities, of course, or even the formation of coordinate singularities that may arise from nonlinearities in the nonprincipal parts of the evolution equations.

Causality is another issue that appears to be less restrictive for our gauge-driver system than it is for directly imposed gauge conditions. For example, the parameter $\nu$ that appears in the $\Gamma$-driver system discussed in Sec. III B must take values in the range $0 \leq \nu \leq \frac{3}{4}$ in order for that $\Gamma$ driver to evolve the shift in a causal way in the BSSN system [25]. There is no such restriction on $\nu$, however, when this $\Gamma$ driver is imposed through our gauge-driver system. In our system the shift is evolved, along with the rest of the spacetime metric, by the GH Einstein system. This system is manifestly hyperbolic, and all of the fields propagate within the physical light cone, no matter what target gauge source function is used in the gauge-driver system.

It is easy to imagine that the system presented here could be improved in several ways. It may be possible, for example, to improve the performance of the system by formulating boundary conditions for $H_{a}$ that impose the desired gauge condition $H_{a}=F_{a}$ exactly at the boundaries. It may also be possible to formulate a different evolution operator for $H_{a}$ that drives it more stably and/or more efficiently toward the desired target $F_{a}$. Finally it may be possible to find better target gauge conditions $F_{a}$. The ones studied here are those which have been found useful in evolutions of traditional three-plus-one formulations of the Einstein system like BSSN. But there may exist gauge conditions having much better stability and effectiveness properties when used as target gauge conditions within a gauge-driver system. These questions, and others, will be addressed in future work on this problem.

\section{ACKNOWLEDGMENTS}

We thank Harald Pfeiffer and Bela Szilagyi for helpful comments concerning this work. The numerical simulations presented here were performed using the Spectral Einstein Code (SpEC) developed at Caltech and Cornell primarily by Larry Kidder, Harald Pfeiffer, and Mark Scheel. This work was supported in part by grants from the Sherman Fairchild Foundation and the Brinson Foundation, by NSF Grant Nos. DMS-0553302, PHY0601459, PHY-0652995, and by NASA Grant No. NNG05GG52G.
[1] F. Pretorius, Classical Quantum Gravity 22, 425 (2005).

[2] F. Pretorius, Phys. Rev. Lett. 95, 121101 (2005).

[3] F. Pretorius, Classical Quantum Gravity 23, S529 (2006).

[4] L. Lindblom, M. A. Scheel, L. E. Kidder, R. Owen, and O.
Rinne, Classical Quantum Gravity 23, S447 (2006).

[5] M. Holst, L. Lindblom, R. Owen, H. P. Pfeiffer, M. A. Scheel, and L. E. Kidder, Phys. Rev. D 70, 084017 (2004).

[6] L. Smarr and J. W. York, Phys. Rev. D 17, 2529 (1978). 
[7] C. Bona, J. Massó, E. Seidel, and J. Stela, Phys. Rev. Lett. 75, 600 (1995).

[8] J. Balakrishna, G. Daues, E. Seidel, W.-M. Suen, M. Tobias, and E. Wang, Classical Quantum Gravity 13, L135 (1996).

[9] M. Alcubierre, B. Brügmann, P. Diener, M. Koppitz, D. Pollney, E. Seidel, and R. Takahashi, Phys. Rev. D 67, 084023 (2003).

[10] M. Campanelli, C. O. Lousto, P. Marronetti, and Y. Zlochower, Phys. Rev. Lett. 96, 111101 (2006).

[11] J. G. Baker, J. Centrella, D.-I. Choi, M. Koppitz, and J. van Meter, Phys. Rev. Lett. 96, 111102 (2006).

[12] T. W. Baumgarte and S. L. Shapiro, Phys. Rev. D 59, 024007 (1998).

[13] L. E. Kidder, L. Lindblom, M. A. Scheel, L. T. Buchman, and H. P. Pfeiffer, Phys. Rev. D 71, 064020 (2005).

[14] M. Boyle, L. Lindblom, H. P. Pfeiffer, M. A. Scheel, and L. E. Kidder, Phys. Rev. D 75,024006 (2007).

[15] J. R. Cash and A. H. Karp, ACM Trans. Math. Softw. 16,
201 (1990).

[16] F. Estabrook, H. Wahlquist, S. Christensen, B. DeWitt, L. Smarr, and E. Tsiang, Phys. Rev. D 7, 2814 (1973).

[17] G. B. Cook and H. P. Pfeiffer, Phys. Rev. D 70, 104016 (2004).

[18] O. Rinne, L. Lindblom, and M.A. Scheel, Classical Quantum Gravity 24, 4053 (2007).

[19] S. A. Teukolsky, Phys. Rev. D 26, 745 (1982).

[20] H. P. Pfeiffer, L.E. Kidder, M. A. Scheel, and D. Shoemaker, Phys. Rev. D 71, 024020 (2005).

[21] M. Alcubierre, Phys. Rev. D 55, 5981 (1997).

[22] M. Alcubierre and J. Massó, Phys. Rev. D 57, R4511 (1998).

[23] M. Alcubierre, Classical Quantum Gravity 22, 4071 (2005).

[24] T. P. Liu, J. Diff. Equ. 33, 92 (1979).

[25] M. Alcubierre, B. Brügmann, P. Diener, M. Koppitz, D. Pollney, E. Seidel, and R. Takahashi, Phys. Rev. D 67, 084023 (2003). 